This item was submitted to Loughborough's Research Repository by the author.

Items in Figshare are protected by copyright, with all rights reserved, unless otherwise indicated.

\title{
Automatic extraction of retinal features from colour retinal images for glaucoma diagnosis: a review
}

PLEASE CITE THE PUBLISHED VERSION

http://dx.doi.org/10.1016/j.compmedimag.2013.09.005

\section{PUBLISHER}

(C) Elsevier

\section{VERSION}

AM (Accepted Manuscript)

\section{PUBLISHER STATEMENT}

This work is made available according to the conditions of the Creative Commons Attribution-NonCommercialNoDerivatives 4.0 International (CC BY-NC-ND 4.0) licence. Full details of this licence are available at: https://creativecommons.org/licenses/by-nc-nd/4.0/

\section{LICENCE}

CC BY-NC-ND 4.0

\section{REPOSITORY RECORD}

Haleem, Muhammad Salman, Liangxiu Han, Jano van Hemert, and Baihua Li. 2019. "Automatic Extraction of Retinal Features from Colour Retinal Images for Glaucoma Diagnosis: A Review”. figshare. https://hdl.handle.net/2134/20268. 


\title{
Automatic extraction of retinal features from colour retinal images for glaucoma diagnosis: A review
}

\author{
Muhammad Salman Haleem ${ }^{\mathrm{a}, *}$, Liangxiu Han ${ }^{\mathrm{a}}$, Jano van Hemert ${ }^{\mathrm{b}}$, Baihua Li $^{\mathrm{a}}$ \\ a School of Computing, Mathematics and Digital Technology, Manchester Metropolitan University, Chester Street, Manchester M1 5GD, United Kingdom \\ ${ }^{\mathrm{b}}$ Queensferry House, Carnegie Business Campus, Enterprise Way, Dunfermline, Scotland KY11 8GR, United Kingdom
}

A B S T R A C T

Glaucoma is a group of eye diseases that have common traits such as, high eye pressure, damage to the Optic Nerve Head and gradual vision loss. It affects peripheral vision and eventually leads to blindness if left untreated. The current common methods of pre-diagnosis of Glaucoma include measurement of Intra-Ocular Pressure (IOP) using Tonometer, Pachymetry, Gonioscopy; which are performed manually by the clinicians. These tests are usually followed by Optic Nerve Head $(\mathrm{ONH})$ Appearance examination for the confirmed diagnosis of Glaucoma. The diagnoses require regular monitoring, which is costly and time consuming. The accuracy and reliability of diagnosis is limited by the domain knowledge of different ophthalmologists. Therefore automatic diagnosis of Glaucoma attracts a lot of attention.

This paper surveys the state-of-the-art of automatic extraction of anatomical features from retinal images to assist early diagnosis of the Glaucoma. We have conducted critical evaluation of the existing automatic extraction methods based on features including Optic Cup to Disc Ratio (CDR), Retinal Nerve Fibre Layer (RNFL), Peripapillary Atrophy (PPA), Neuroretinal Rim Notching, Vasculature Shift, etc., which adds value on efficient feature extraction related to Glaucoma diagnosis. 


\section{Introduction}

Early detection and treatment of retinal eye diseases is critical to avoid preventable vision loss. Conventionally, retinal disease identification techniques are based on manual observations. Patients are imaged using a fundus camera or Scanning Laser Ophthalmoscope (SLO). Optometrists and ophthalmologists often relay on image operations such as change of contrast and zooming to interpret these images and diagnose results based on their own experience and domain knowledge. If specific abnormalities are observed, ophthalmologists may perform Fluorescein Angiography or Optical Coherence Tomography (OCT) for further investigation. These diagnostic techniques are time consuming and invasive. Automating procedures of diagnosis expect more patients to be screened and more consistent diagnoses can be given in a time efficient manner.

Glaucoma is one of the most common and leading cause of blindness [1] among retinal diseases with $13 \%$ of the cases being affected [2]. The changes occur in retinal structures, which gradually leads to peripheral vision loss and eventually causes blindness if left untreated. There is no cure for Glaucoma but its early diagnosis and treatment can prevent the vision loss. Since the manual based diagnostic process is costly and prone to error, therefore some efforts have been made on automatic detection of Glaucoma at an early stage. In this paper, we have conducted a comprehensive review on existing methods for automatic identification of anatomical features related to Glaucoma from retinal images, which provides insights for future directions on automatic detection of Glaucoma and other eye diseases from retinal images.

The rest of the sections is organized as follows: Section 2 briefly describes Glaucoma and the changes in retinal structures associated with Glaucoma. In Section 3, we discuss the current clinical methods for Glaucoma diagnosis. Section 4 overviews the existing methods on automatic detection of retinal features associated with Glaucoma in a systematic way. Section 7 concludes the work.

\section{Retinal symptoms of glaucoma}

Glaucoma is a group of eye diseases which is associated with simultaneous functional failure of the visual field. The structural changes are manifested by a slowly diminishing neuroretinal rim indicating a degeneration of axons and astrocytes of the Optic Nerve. As lost capabilities of the Optic Nerve cannot be recovered, early detection and subsequent treatment are essential for affected patients to preserve their vision [3].

There are two main types of Glaucoma (i) Primary Open Angle Glaucoma (POAG) and (ii) Angle Closure Glaucoma (ACG). POAG is the most common form of Glaucoma accounting for at least $90 \%$ of all Glaucoma cases [4]. The Intra-Ocular Pressure (IOP), which maintains a permanent shape of the human eye and protects it from deformation, rises because the correct amount of fluid cannot drain out of the eye. With POAG, the entrances to the drainage canals work properly but a clogging problem occurs inside the drainage canals [5]. This type of Glaucoma develops slowly and sometimes without noticeable sight loss for many years. It can be treated with medications if diagnosed at the earlier stage. ACG happens when the drainage canals get blocked. The iris is not as wide and open as in the normal case. The outer edge of the iris bunches up over the drainage canals, when the pupil enlarges too much or too quickly. Treatment of this type of Glaucoma usually involves surgery to remove a small portion of the outer edge of the iris. There are four main changes in the retinal structures which are associated with Glaucoma and are explained as follows:

\subsection{Optic Nerve Head variance}

The Optic Nerve Head (ONH) is the location where the Optic Nerve enters the back of the eye. It is also known as blind spot since this area of retina cannot respond to light stimulation due to lack of photoreceptors. In a typical 2D retinal image, the Optic Nerve Head is a bright elliptic region with a distinguishable cup-like area called Optic Cup surrounded by rest of the area of Optic Disc, as shown in Fig. 1. There are several ways to quantify the Optic Cup to Disc Ratio (CDR) [6]. For instance, the ratio can be defined with respect to area, vertical length and horizontal length of both Optic Disc and Optic Cup. The CDR can be used to compare Glaucoma patients with Normal subjects, and it is an important measurement for the diagnosis of Glaucoma [6]. When more Optic Nerve Fibres disappear, the Optic Cup becomes larger with respect to the Optic Disc, which corresponds to increase in CDR value. In current clinical practice, the CDR is measured manually by an ophthalmologist and is subjective due to differences in the intra-observer experiences and training [7]. 


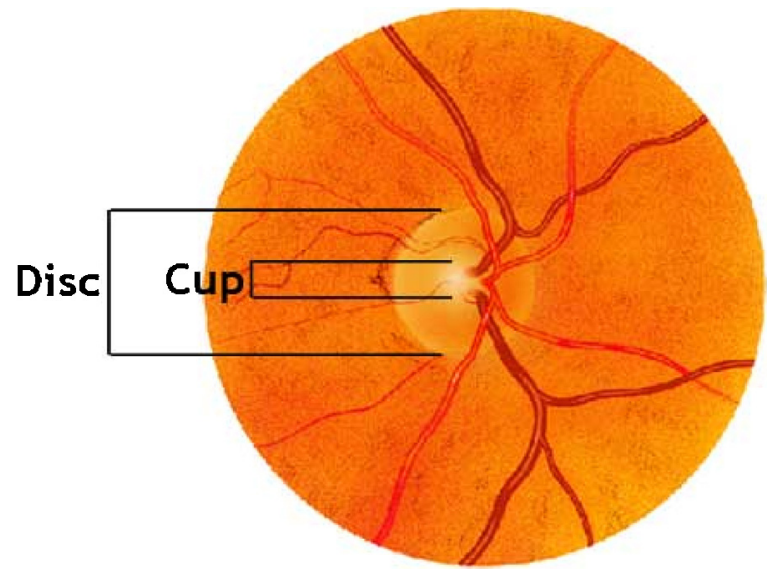

Fig. 1. Optic Disc and Optic Cup in fundus image.

\subsection{Neuroretinal Rim loss determination}

In Normal eyes, the Neuroretinal Rim usually follows a characteristic pattern as shown in retinal image taken from the right eye in Fig. 2. It is the broadest in the Inferior region followed by the Superior, then Nasal and finally the Temporal regions, which is called the ISNT rule [8]. In case of Glaucoma, the Neuroretinal Rim disobeys the earlier mentioned rule. Although this is not the case with all Glaucoma patients [9], it is still a useful clinical method to aid in diagnosing Glaucoma. For the left eye, the position of Temporal and Nasal region will swap each other.

\subsection{Retinal Nerve Fibre Layer (RNFL) defects}

According to clinical reports, RNFL defects, if detected, serve as the earliest sign of Glaucoma [10]. The RNFL appears as bright

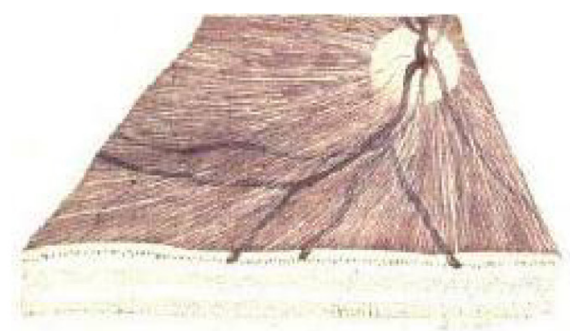

(a)

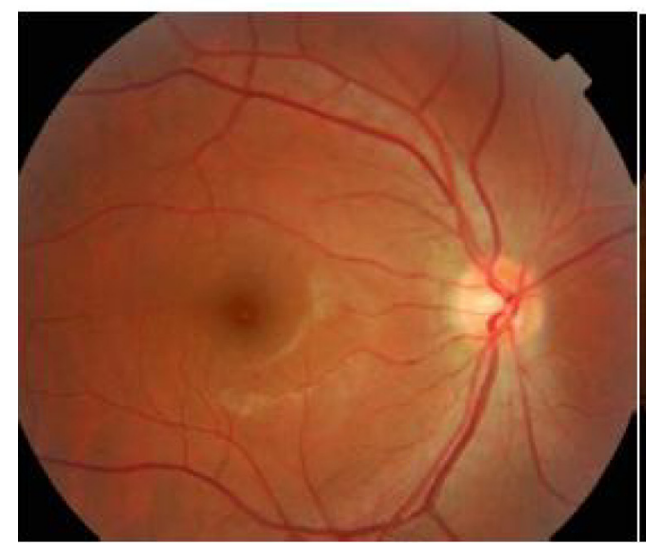

(c)

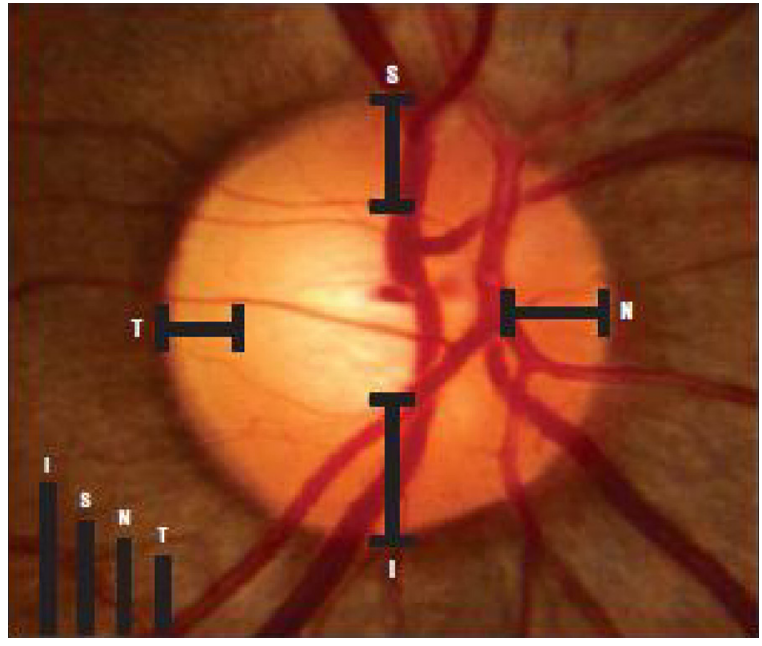

Fig. 2. Clinical assessment based on the ISNT rule obtained from Normal optic nerves. I, S, N and T represent Inferior, Superior, Nasal and Temporal regions separately. The image is taken from the right eye.

bundle striations that are unevenly distributed in Normal eyes. It can be assessed ophthalmoscopically on wide-angle, red-free photographs or by using sophisticated techniques such as Scanning Laser Ophthalmoscope(SLO) and Laser Polarimetry. In Normal eyes, the RNFL striations are most visible in the Temporal Inferior region, followed by the Temporal Superior, the Nasal Superior and the Nasal Inferior regions. There are two types of RNFL defects associated with Glaucomatous eyes. A localised RNFL defect appears as a wedge-shaped dark area in the RNFL with its tip touching the Optic Disc border [10] as shown in Fig. 3. They are often found in the Temporal Inferior region followed by the Temporal Superior

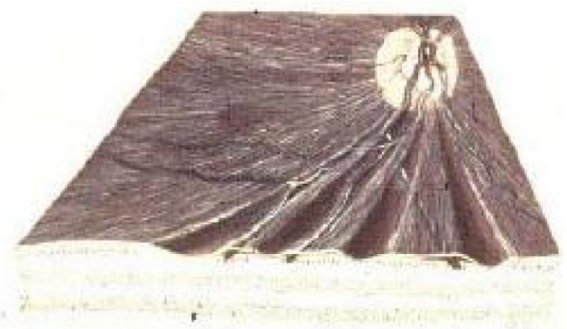

(b)

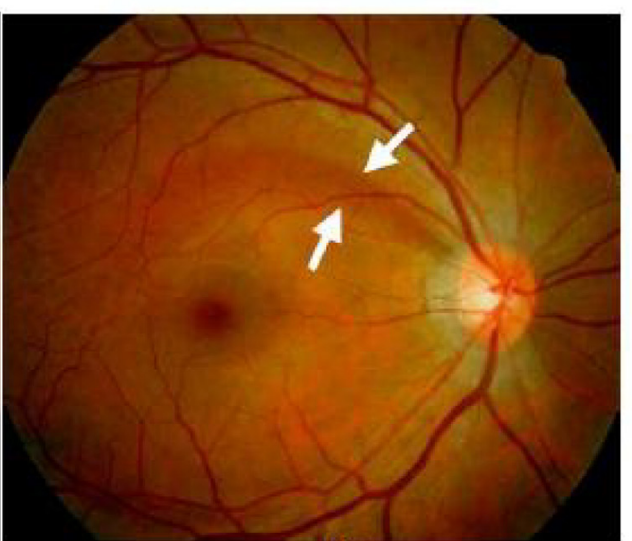

(d)

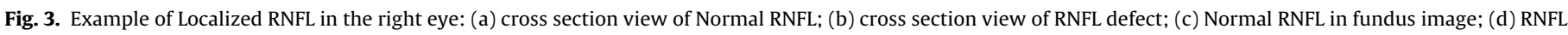
defect in fundus image (white arrows pointed) [11] 

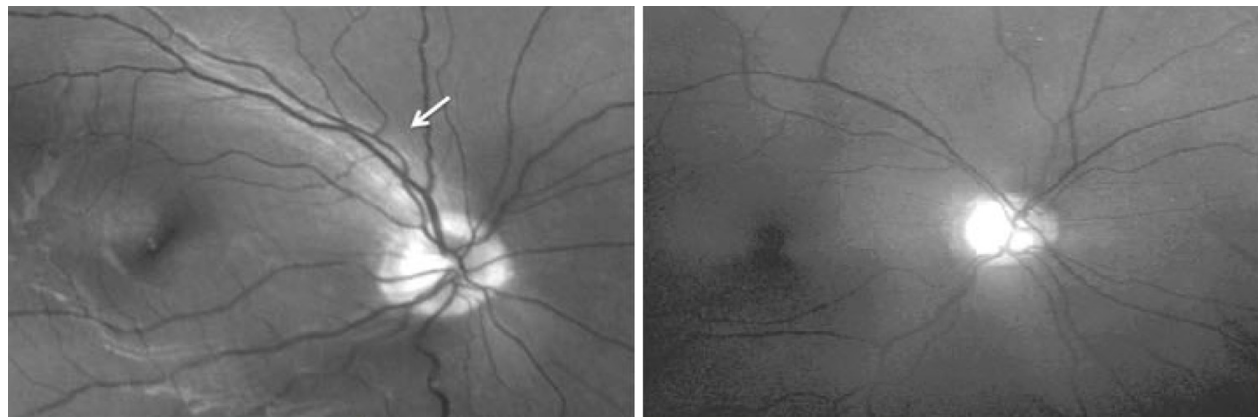

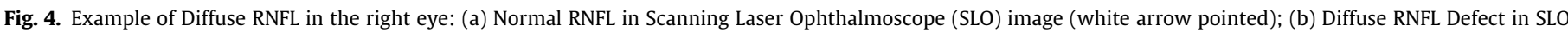
image [12].

region. Diffuse RNFL defect results in a decrease in appearance of RNFL due to loss of ganglion cells as shown in Fig. 4.

\subsection{Peripapillary Atrophy}

Peripapillary Atrophy (PPA) is an important risk factor and its progression can lead to Disc Haemorrhage and thus Glaucoma [13]. It is the degeneration of the retinal pigment epithelial layer, photoreceptors and underlying choriocapillaries in the region surrounding the $\mathrm{ONH}$ [14]. PPA is divided into two zones namely central $\beta$-zone and peripheral $\alpha$-zone as shown in Fig. 5. $\alpha$-zone is characterized by irregular hypopigmentation, hyperpigmentation and intimated thinning of the chorioretinal tissue layer. On its outer side it is adjacent to retina and on its inner side it is in touch with $\beta$-zone characterized by visible sclera and visible large choroidal vessels. In Normal eyes, both $\alpha$-zone and $\beta$-zone are frequently located in the Temporal region followed by Inferior region and Superior region. In Glaucomatous eyes, $\beta$-zone occurs more frequently in the Temporal region [15] and its extent correlates with the RNFL thinning.

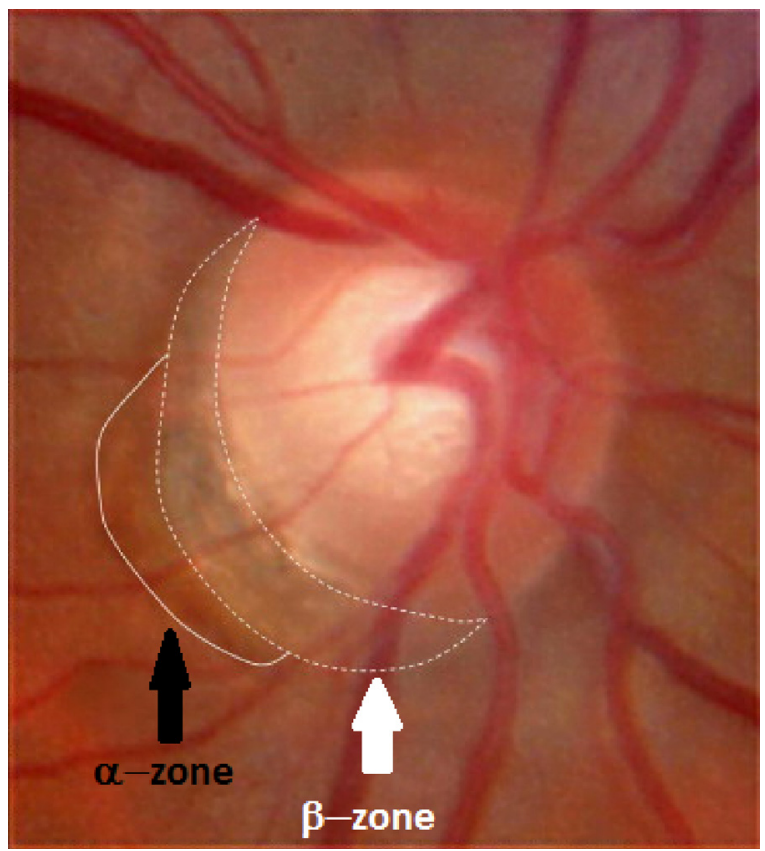

Fig. 5. PPA with $\alpha$-zone and $\beta$-zone [16]. $\beta$-Zone occurs more frequently in the Temporal region of the right eye.

\section{Clinical methods for diagnosing glaucoma}

Glaucoma is usually realized by a patient after a long time of disease progression. This is because Glaucoma usually damages the outer edge of the eye and works slowly inwards [17]. Therefore, it is important to have regular eye tests so that any symptoms can be detected and treated as early as possible. According to UK's National Institute of Health and Clinical Excellence (NICE) guidelines [18], the tests which should be offered for suspecting Glaucoma or Ocular Hypertension are eye pressure test (Tonometry), corneal thickness (Pachymetry), Gonioscopy, visual field test (Perimetry) and $\mathrm{ONH}$ appearance.

Tonometry uses an instrument called a tonometer to measure IOP. Glaucoma is suspected if IOP value exceeds $21 \mathrm{Hg}$. There are several techniques for tonometry. Among those techniques, Goldmann Applanation is considered the gold standard among other tests [19] as inter and intra-observer variability is between 2-3 $\mathrm{mmHg}$. However, high pressures of IOP are not an accurate indication for Glaucoma [20] as different patients have different cornea thickness. Therefore, Pachymetry is the test used to determine thickness of cornea and it is important because actual IOP may be underestimated in the patients with thinner cornea, and overestimated in the patients with thicker cornea [21]. Gonioscopy can help to determine whether this angle is open or closed (blocked). It is an examination of the front outer edge of the eye, between the cornea and the iris. This is the area where the fluid should drain out of the eye. As peripheral vision is usually the first to deteriorate in Glaucoma, Perimetry is a systematic measurement of light sensitivity in the visual field by detection of targets presented on defined background. It checks for missing areas of peripheral vision. It may take three examinations before an accurate baseline is obtained and moreover long-term fluctuations in the field tests can often occur. Therefore the accuracy of this method is still questioned [22,23].

Glaucoma leads to alterations in the $\mathrm{ONH}$, neuroretinal rim and vasculature structure. By analyzing retinal images, anatomical changes in retina due to Glaucoma can be observed. However, manual observation of $\mathrm{ONH}$ is time consuming and subject to inter-observer variability. Therefore efforts are being conducted to determine the anatomical changes in the retinal structures due to Glaucoma from the retinal images automatically. Also because changes observed in RNFL are also one of the early signs of Glaucoma, ophthalmologists usually go for $3 \mathrm{D}$ imaging in order to determine the thickness of RNFL. These imaging modalities include Scanning Laser Polarimetry (SLP), Confocal Scanning Laser Ophthalmoscopy (CSLO) and Optical Coherence Tomography (OCT) [24]. SLP utilizes the birefringent properties of the RNFL to measure its thickness by measuring the phase-shift of the polarized light through the layer. CSLO employs the principle of light reflection. Here a laser is projected through a pinhole towards the area of interest and the reflections are picked up through another pinhole 
in front of light detector. OCT, based upon interferometry, is a noninvasive, non-contact method of producing high-resolution images utilizing coherence property of light reflected from a scanned area [25]. Its working principle is like ultrasound but OCT has higher resolution and it discriminates quite well between Normal and Glaucomatous eyes. Nevertheless, its role in detecting any Glaucomatous progression is uncertain [26].

\section{Segmentation based automatic feature detection methods of retinal structures symptomatic for glaucoma}

Since the current clinical methods for the diagnosis of Glaucoma are mostly based on manual observations and sometimes invasive, therefore the use of automatic detection of features related to Glaucoma can aid their diagnosis in a time effective and non-invasive manner. There is no cure for Glaucoma but its diagnosis and treatment at earlier stage can slow down the progression of the disease. There are some segmentation and non-segmentation (discussed in the Section 5) based automatic methods used to determine features of the retinal structures that reflect changes due to Glaucoma. Since most of segmentation based need $\mathrm{ONH}$ analysis, therefore, most of the existing methods for analysis of the Glaucoma are related to extraction of $\mathrm{ONH}$ and its anatomical structures. Still, there are some methods of detecting RNFL defect which aid in diagnosing Glaucoma and do not require Optic Disc detection and segmentation. The methods described in this section are classified in terms of image processing techniques and are mentioned in the form of hierarchy as shown in Fig. 6. Their results are summarized in Tables 1-5. The results which were applied on common datasets are grouped together so as to compare different methods on a common benchmark. Moreover the evaluation measures used in Literature Survey are defined in A and the public databases used for experimentation are briefed in $\mathrm{B}$.

\subsection{Localization of $\mathrm{ONH}$}

The Localization of ONH is the initial step in order to locate other anatomical structures, vessel tracking and registering changes within Optic Disc region. If any pixel within the Optic Disc boundary is located, then it can facilitate the extraction of the Optic Disc boundary. There are three main categories for the localization methods of the Optic Disc boundary which are discussed in the following paragraphs and a summary of the methods is shown in Table 1.

\subsubsection{Optic disc detection as the brightest region}

The first category is based on calculating the intensity of pixels in coloured and grayscale image. Under normal circumstances, the Optic Disc region is the brightest region on the retinal image. Therefore it can be detected by thresholding out the pixels with the intensity values below the certain level [27].

Sinthanayothin et al. [28] localized the Optic Disc using Local Contrast Colour Enhancement in Intensity-Hue-Saturation space. The variance image after converting back to RGB space will intensify the Optic Disc as compared to rest of the image. The algorithm resulted in high accuracy in their local dataset however, others have found its high failure rate in the images with a large number of white lesions, light artifacts or strongly visible choroidal vessels [29]. The use of pre-processing steps such as image filtering for removing artifacts brighter than the Optic Disc may result in improvement of the results. Walter et al. [30] localized the Optic Disc center with the proposal to select the brightest two percent of the image pixels to determine a threshold. After thresholding the image, the region with the largest area is likely to be Optic Disc region. The algorithm was applied on a set of 30 retinal images however, it failed on a low contrast image. Sekhar et al. [31] improved the method by utilizing mathematical morphology to apply the shade correction on the retinal image. The mathematical morphology is the process of analyzing the geometrical structures present in the image by affecting their shape and form [32]. The largest area of the shape corrected retinal image was considered as Optic Disc region. The shade correction although improved the localization accuracy as compared to the previous method, its role in locating the Optic Disc region is uncertain. In order to locate the Optic Disc automatically, both methods used Circular Hough Transform [33] which aims to find circular patterns within an image.

In spite of assuming the Optic Disc as the brightest region of the retinal image is the straightforward approach to localize the region, the method is unreliable. The technique usually fails to localize the Optic Disc in the retinal images having artifacts and the bright regions in the diseased retina. Moreover, this assumption is not a guaranteed way to determine the center of the Optic Disc.

\subsubsection{Center localization by matching of the Optic Disc template}

The second category is based on formation of template representing the Optic Disc and matching the template in a test image. The results were comparatively accurate as compared to first category at the expense of the computational time. Later on, the computational time was reduced by introducing efficient algorithmic procedure.

Li et al. [40] modeled the Optic Disc texture using Principal Component Analysis (PCA). The Optic Disc in a test image was localized by dividing the test image into sub-images and Euclidean distance from the PCA-model was calculated for each sub-image. The subimage with the Optic Disc at the center will have least Euclidean distance from the PCA model. The results were accurate but the search procedure was computationally inefficient. Computational time may be reduced if the image resolution is reduced as less number of pixels will allow to execute the task earlier but consequently, it might skip some of the textural information resulting in decrease in accuracy of the localization. Osareh et al. [37] evaluated the normalized correlation coefficient in order to compare the template image and the region of consideration. The template was generated after averaging the 16 colour-normalized retinal images. The method only detected the location based on highest value of normalized correlation coefficient which does not necessarily mean the Optic Disc center. They further improved their template by dividing the Optic Disc into smaller segments and selecting the largest segment which is approximate to a half circle and the region is not occluded by blood vessels. The algorithm followed the iterative procedure for minimizing the error between the set of pixels in the arc points and the estimated arc. The center of the circular arc is the approximate location of Optic Disc center. These template based matching algorithms were less computationally efficient therefore some pre-processing steps were introduced in order to improve the computational time.

Lalonde et al. [38] proposed an Optic Disc center detection method based on Pyramidal Decomposition and circular template matching. Pyramidal Decomposition reduces the image resolution to one-fourth at each iteration level. At the fifth level, bright regions of retina other than Optic Disc are vanished. The localization of the brightest pixel led to the determination of Optic Disc area for determining the edges of anatomical structures. The edge map was thresholded based on the strongest edges in the retinal image and the circular template was then compared along the pixels having strong edges. This made the computational time smaller as the template did not need to be compared pixel by pixel in the retinal image. Then pixel with the lowest degree of mismatch (Hausdorff distance) with the circular template and the highest confidence value was regarded as Optic Disc center. The author reported the failure cases when pyramidal decomposition sometimes localized the brightest pixel very far from Optic Disc due to artifacts in the retinal 
Table 1

Accuracy of several methods of optic disc detection/center localization.

\begin{tabular}{|c|c|c|c|c|c|c|}
\hline Localization method & Image database & Colour space & $\begin{array}{l}\text { Image } \\
\text { dimension }\end{array}$ & $\begin{array}{l}\text { Number of } \\
\text { test images }\end{array}$ & Accuracy & Citation \\
\hline \multicolumn{7}{|l|}{ ONH as brightest region } \\
\hline Local Contrast Colour Enhancement & STARE [34] & $\begin{array}{l}\text { 24-Bit RGB to } \\
\text { IHS space }\end{array}$ & $605 \times 700$ & 81 & $42 \%$ within $\mathrm{ONH}$ & {$[28,35]$} \\
\hline Thresholding Highest Pixel Intensities & STARE [34] & $\begin{array}{l}\text { 24-Bit RGB to } \\
\text { HLS space }\end{array}$ & $605 \times 700$ & 81 & $58 \%$ within $\mathrm{ONH}$ & {$[30,35]$} \\
\hline \multirow[t]{2}{*}{ Shade Correction using Morphology } & STARE [34] & 24-Bit RGB & $605 \times 700$ & 17 & $82.3 \%$ within $\mathrm{ONH}$ & \multirow[t]{2}{*}{ [31] } \\
\hline & DRIVE [36] & 8-Bit RGB & $768 \times 584$ & 38 & $94.7 \%$ within $\mathrm{ONH}$ & \\
\hline \multicolumn{7}{|l|}{ ONH template matching } \\
\hline Least Squares Regression Arc Estimation & STARE [34] & 24-Bit RGB & $605 \times 700$ & 81 & $58 \%$ within $\mathrm{ONH}$ & {$[37,35]$} \\
\hline Pyramidal Decomposition and & STARE [34] & 24-Bit RGB & $605 \times 700$ & 81 & $71.6 \%$ within & {$[38,35]$} \\
\hline Circular Template Matching & & & & & $\mathrm{ONH}$ & \\
\hline \multirow[t]{2}{*}{ Vasculature Detection using matched filter } & STARE [34] & 24-Bit RGB & $605 \times 700$ & 81 & $98.7 \%$ within 60 pixels & \multirow[t]{2}{*}{ [39] } \\
\hline & DRIVE [36] & 8-Bit RGB & $768 \times 584$ & 40 & $100 \%$ within 60 pixels & \\
\hline Principal Component Analysis & $\begin{array}{l}\text { National University } \\
\text { Hospital Singapore }\end{array}$ & 24-Bit RGB & $512 \times 512$ & 40 & - & [40] \\
\hline Averaging Optic Disc Region & Bristol Eye Hospital & 24-Bit RGB & $760 \times 570$ & 60 & - & [37] \\
\hline \multicolumn{7}{|l|}{ Vasculature convergence point } \\
\hline Fuzzy Convergence of Blood Vessels & STARE [34] & 24-Bit RGB & $605 \times 700$ & 81 & $89 \%$ within 60 pixels & [41] \\
\hline Mathematical modeling of Vasculature Structure & STARE [34] & 24-Bit RGB & $605 \times 700$ & 81 & $98 \%$ within $\mathrm{ONH}$ & {$[42]$} \\
\hline Tensor Voting and Mean-Shift procedure & STARE [34] & 24-Bit RGB & $605 \times 700$ & 81 & 92\% within $\mathrm{ONH}$ & [43] \\
\hline \multirow[t]{2}{*}{ Horizontal and Vertical Edge Mapping } & STARE [34] & 24-Bit RGB & $605 \times 700$ & 81 & \multirow[t]{2}{*}{$97 \%$ within 60 pixels } & \multirow[t]{2}{*}[44]{} \\
\hline & DRIVE [36] & 8-Bit RGB & $768 \times 584$ & 40 & & \\
\hline Fractal Dimension Analysis & DRIVE [36] & 8-Bit RGB & $768 \times 584$ & 40 & - & [45] \\
\hline \multirow[t]{3}{*}{ Gabor Filter Vessel Detection } & MESSIDOR [46] & 8-Bit RGB to & $1440 \times 960$ & 1200 & $98.3 \%$ within $\mathrm{ONH}$ & [47] \\
\hline & & CIE space & $2240 \times 1488$ & & & \\
\hline & & & $2304 \times 1536$ & & & \\
\hline
\end{tabular}

Table 2

Results of different methods of optic disc extraction.

\begin{tabular}{|c|c|c|c|c|c|c|}
\hline Localization method & Image database & Colour space & Image dimension & $\begin{array}{l}\text { Number of test } \\
\text { images }\end{array}$ & Accuracy & Citation \\
\hline \multicolumn{7}{|l|}{ Non-model based approach } \\
\hline Morphological Operation & $\begin{array}{l}\text { Kasturba Medical College } \\
\text { India }\end{array}$ & 8-Bit RGB to grayscale & $560 \times 720$ & $\begin{array}{l}24 \text { Normal and } \\
37 \text { Glaucoma }\end{array}$ & $\begin{array}{l}\text { 90\% Neural Network } \\
\text { [49] classification }\end{array}$ & [48] \\
\hline Fuzzy Histogram & $\begin{array}{l}\text { Kasturba Medical College } \\
\text { India }\end{array}$ & 8-Bit RGB to Lab space & $560 \times 720$ & $\begin{array}{l}30 \text { Normal, } 31 \\
\text { Retinopathy and } \\
39 \text { Glaucoma }\end{array}$ & $\begin{array}{l}93.4 \% \text { Overlapping } \\
\text { Score }\end{array}$ & [50] \\
\hline $\begin{array}{l}\text { Circular Hough Transform on } \\
\text { gradient map }\end{array}$ & MESSIDOR [46] & 8-Bit RGB & $\begin{array}{r}1440 \times 960 \\
2240 \times 1488 \\
2304 \times 1536\end{array}$ & 1200 & $86 \%$ Overlapping Score & [51] \\
\hline $\begin{array}{l}\text { Genetic Algorithm for elliptical } \\
\text { approximation }\end{array}$ & DRION-DB [52] & 8-Bit RGB & $600 \times 400$ & 110 & $\begin{array}{l}96 \% \text { images have } \leq 5 \\
\text { pixel MAD }\end{array}$ & [52] \\
\hline K-Means Clustering & Aravind Eye Hospital India & YUV space & $1504 \times 1000$ & $\begin{array}{l}20 \text { Normal and } \\
25 \text { Glaucoma }\end{array}$ & $\begin{array}{l}\text { 91.7\% CDR Correlation } \\
\text { Coefficient }\end{array}$ & {$[53]$} \\
\hline Watershed Transformation & Mines ParisTech & 8-Bit RGB to HLS space & $640 \times 480$ & 30 & - & [30] \\
\hline Hough Transform on Edge Map & DRIVE [36] & 8-Bit RGB to Y channel & $768 \times 584$ & 40 & 73\% Overlapping Score & [54] \\
\hline Thresholding & STARE [34] & 24-Bit RGB & $605 \times 700$ & 81 & $93 \%$ of OD diameter & [55] \\
\hline \multicolumn{7}{|l|}{ Freeform modeling } \\
\hline Morphological Preprocessing & New York Library & 8-Bit RGB to YIQ space & $285 \times 400$ & 9 & - & {$[56,58]$} \\
\hline Morphological Preprocessing & Bristol Hospital & 24-Bit RGB to Lab space & $760 \times 570$ & 16 & $\begin{array}{l}\text { 90.3\% Overlapping } \\
\text { Score }\end{array}$ & {$[58]$} \\
\hline $\begin{array}{l}\text { Circular Hough Transform } \\
\text { Initialization }\end{array}$ & $\begin{array}{l}\text { National University } \\
\text { Hospital Singapore }\end{array}$ & 24-Bit RGB have $\leq 3$ pixel & $512 \times 512$ & 100 & 93\% images MAD & [59] \\
\hline Level Set Approach & Aravind Eye Hospital India & 8-Bit RGB & $2896 \times 1944$ & $\begin{array}{l}33 \text { Normal and } \\
105 \text { Glaucoma }\end{array}$ & 97\% Overlapping Score & {$[60]$} \\
\hline Fast Level-Set Approach & MESSIDOR [46] & 8-Bit RGB & $\begin{array}{r}1440 \times 960 \\
2240 \times 1488 \\
2304 \times 1536\end{array}$ & 1200 & $\begin{array}{l}89.5 \% 1-(\mathrm{MAD} / \mathrm{OD} \\
\text { radius })\end{array}$ & [47] \\
\hline \multicolumn{7}{|l|}{ Statistical shape modeling } \\
\hline $\begin{array}{l}\text { Image Gradient Based } \\
\text { Landmark Search }\end{array}$ & $\begin{array}{l}\text { National University } \\
\text { Hospital Singapore }\end{array}$ & 24-Bit RGB & $512 \times 512$ & 35 & $\begin{array}{l}82 \% \text { images have } \leq 3 \\
\text { pixel MAD }\end{array}$ & [61] \\
\hline RANSAC approach & Seoul University Korea & 8-Bit RGB & $2896 \times 1944$ & $\begin{array}{l}53 \text { Normal and } \\
30 \text { Glaucoma }\end{array}$ & $\begin{array}{l}84.5 \% \text { Overlapping } \\
\text { Score }\end{array}$ & [62] \\
\hline $\begin{array}{l}\text { Minimum Mahalanobis } \\
\text { distance Method }\end{array}$ & ORIGA-light $[63]$ & 8-Bit RGB & $3072 \times 2048$ & $\begin{array}{l}482 \text { Normal and } \\
168 \text { Glaucoma }\end{array}$ & 89\% Overlapping Score & [11] \\
\hline $\begin{array}{l}\text { Search Space Restriction along } \\
\text { Normal Lines }\end{array}$ & DRION-DB [52] & 8-Bit RGB & $600 \times 400$ & 110 & 92\% Overlapping Score & [65] \\
\hline
\end{tabular}




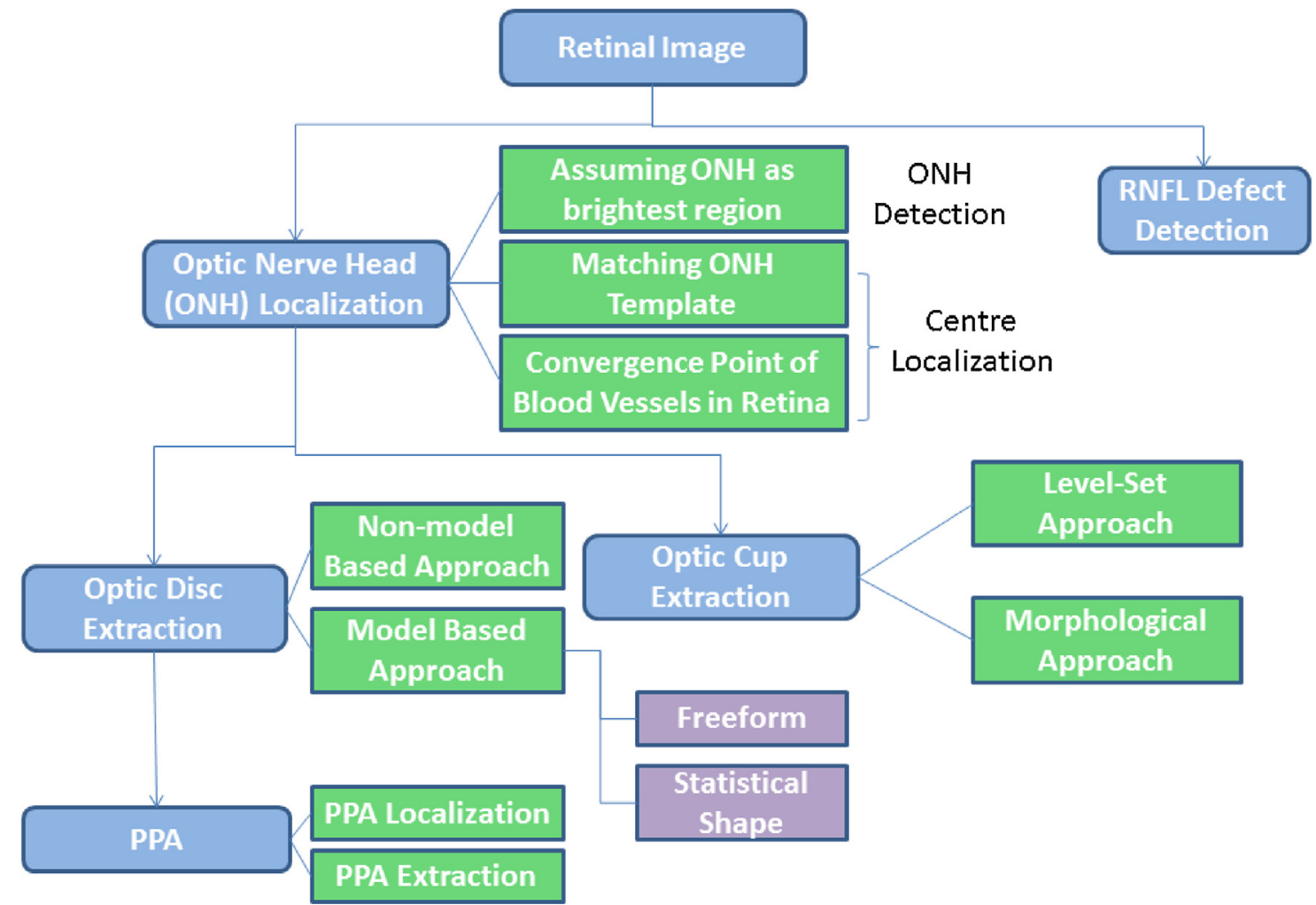

Fig. 6. A hierarchy of methodologies for segmentation based automatic feature extraction from digital retinal images.

Table 3

Results of different methods of optic cup extraction.

\begin{tabular}{|c|c|c|c|c|c|c|}
\hline Localization method & Image database & Colour space & Image dimension & $\begin{array}{l}\text { Number of test } \\
\text { images }\end{array}$ & Accuracy & Citation \\
\hline \multicolumn{7}{|l|}{ Morphological approach } \\
\hline Fuzzy C-means Clustering & $\begin{array}{l}\text { Aravind Eye } \\
\text { Hospital India }\end{array}$ & RGB space & $1504 \times 1000$ & $\begin{array}{l}20 \text { Normal and } 25 \\
\text { Glaucoma }\end{array}$ & $\begin{array}{l}91.7 \% \text { CDR } \\
\text { Correlation } \\
\text { Coefficient }\end{array}$ & {$[53]$} \\
\hline Morphological Operations & $\begin{array}{l}\text { Kasturba Medical } \\
\text { College India }\end{array}$ & $\begin{array}{l}\text { 8-Bit RGB } \\
\text { converted to } \\
\text { grayscale }\end{array}$ & $560 \times 720$ & $\begin{array}{l}24 \text { Normal and } 37 \\
\text { Glaucoma }\end{array}$ & $\begin{array}{l}90 \% \text { Neural } \\
\text { Network [49] } \\
\text { classification }\end{array}$ & {$[48]$} \\
\hline \multicolumn{7}{|l|}{ Level-set approach } \\
\hline Vessel Kink Interpolation in 3D & $\begin{array}{l}\text { Aravind Eye } \\
\text { Hospital India }\end{array}$ & 8-Bit RGB & $2896 \times 1944$ & $\begin{array}{l}33 \text { Normal and } 105 \\
\text { Glaucoma }\end{array}$ & $\begin{array}{l}82 \% \text { Overlapping } \\
\text { Score }\end{array}$ & {$[60]$} \\
\hline Gradient Vector Flow Modeling & ORIGA $^{\text {-light }}[63]$ & 8-Bit RGB & $3072 \times 2048$ & $\begin{array}{l}94 \text { Normal and } 10 \\
\text { Glaucoma }\end{array}$ & $\begin{array}{l}\text { 90.85\% CDR } \\
\text { Correlation } \\
\text { Coefficient }\end{array}$ & [73] \\
\hline Blood Vessel Kinks Edge Detection & ORIGA ${ }^{-l i g h t}[63]$ & 8-Bit RGB & $3072 \times 2048$ & $\begin{array}{l}17 \text { Normal and } 10 \\
\text { Glaucoma }\end{array}$ & $\begin{array}{l}\text { 95.2\% CDR } \\
\text { Correlation } \\
\text { Coefficient }\end{array}$ & [74] \\
\hline
\end{tabular}

Table 4

Results of different methods of PPA localization/extraction.

\begin{tabular}{|c|c|c|c|c|c|c|}
\hline Extraction method & Image database & Colour space & Image dimension & $\begin{array}{l}\text { Number of test } \\
\text { images }\end{array}$ & Accuracy & Citation \\
\hline \multicolumn{7}{|l|}{ PPA detection } \\
\hline Disc Difference Method & SCORM [75] & RGB converted to HSV space & $800 \times 800$ & $\begin{array}{l}20 \text { Normal and } 20 \\
\text { Glaucoma }\end{array}$ & 95\% within PPA & [76] \\
\hline GLCM Based Texture Analysis & SCORM [75] & RGB converted to HSV space & $800 \times 800$ & $\begin{array}{l}20 \text { Normal and } 20 \\
\text { Glaucoma }\end{array}$ & 92.5\% within PPA & [77] \\
\hline \multicolumn{7}{|l|}{ PPA extraction } \\
\hline GLCM Based Texture Analysis & $\begin{array}{l}\text { Gifu University } \\
\text { Hospital Japan }\end{array}$ & 8-Bit RGB & $1600 \times 1200$ & 26 & $\begin{array}{l}\text { 73\% LDA Segmentation } \\
\text { Overlapping Score }\end{array}$ & [78] \\
\hline
\end{tabular}


Table 5

Results of different methods of RNFL detection.

\begin{tabular}{|c|c|c|c|c|c|c|}
\hline Extraction method & Image database & Colour space & Image dimensions & Number of test images & Accuracy & Citation \\
\hline Directional Gabor Filters & $\begin{array}{l}\text { Gifu University } \\
\text { Japan }\end{array}$ & $\begin{array}{l}\text { RGB space } \\
\text { converted to } \\
\text { grayscale }\end{array}$ & $768 \times 576$ & 52 & $71 \%$ & [81] \\
\hline Intensity Profile Plotting & $\begin{array}{l}\text { Regional Institute } \\
\text { of Ophthalmology, } \\
\text { India }\end{array}$ & Green plane of RGB & $768 \times 576$ & $\begin{array}{l}300 \text { Normal and } 529 \\
\text { Glaucoma }\end{array}$ & $91.5 \%$ & {$[82]$} \\
\hline Markov Random Fields & $\begin{array}{l}\text { Eye Clinic Zlin, } \\
\text { Czech Rep }\end{array}$ & $\begin{array}{l}\text { Green and Blue } \\
\text { Channels of RGB } \\
\text { space }\end{array}$ & $3504 \times 2336$ & $\begin{array}{l}18 \text { Normal and } 10 \\
\text { Glaucoma }\end{array}$ & $89.3 \%$ & {$[83,84]$} \\
\hline
\end{tabular}

image, and Hausdorff distance based circular template matching was failed when Optic Disc was highly occluded by blood vessels. As discussed earlier, the use of image filtering e.g. Directional Gabor Filters as a preprocessing step can improve the results. Yousiff et al. [39] applied directional matched filter in order to determine the vasculature structure. Then a window of 9x9 from the test image was compared on the pixels belonging to the vasculature structure which can be resized based on size of the image. The pixel with least accumulative distance was regarded as Optic Disc center. Yu et al. [47] used binary template to define the search region for the Optic Disc. They further applied the Directional Gabor Filters and calculated the standard deviation of the Optic Disc pixel candidates. The pixel candidates were determined after sorting the pixels of the search region using Pearson's correlation coefficient. The pixel candidates with maximum standard deviation was regarded as Optic Disc center.

Center localization of the Optic Disc using template matching proved to be more accurate as compared to the methods based on assumption of highest pixel intensity values within Optic Disc region due to non-uniform illumination in the retinal image. PCA and circular arc based template matching were the initial steps towards the Optic Disc center localization. In spite of they were more accurate as compared to pixel intensity based techniques, they were still not able to distinguish among artifacts across the retinal image. Moreover, the removal of blood vessel occlusion reduced the quality of template to be compared. Pyramidal Decomposition improved the center detection in terms of computational time however, the misguidance by artifacts remained. The use of directional filters for vasculature segmentation were then introduced which not only reduced the search region for the best candidate of the Optic Disc center but also improved the detection accuracy. But the use of directional filters may be less applicable to the images diseased with glaucoma as the atrophy around the Optic Disc would also be segmented out as the part of the vasculature structure which may affect the detection accuracy based on template matching.

\subsubsection{ONH center as convergence point of retinal blood vessels}

The third category regards the convergence point of blood vessels in retina to be the center of the ONH. This assumption avoided mis-localization of the Optic Disc as most of the retinal blood vessels converge within the $\mathrm{ONH}$ region. Moreover if the retinal vasculature structure is segmented out accurately, it can also avoid the detection failure of Optic Disc center due to template matching.

Hoover et al. [41] segmented out the blood vessels in order to determine the point of intersection. A voting process was introduced in order to find out points where a large number of blood vessels combine and the point with maximum votes was regarded as ONH center. Then, illumination equalization was applied in order to determine the different region in retinal image as the method was also applied to retinal images having diseases such as Diabetic Retinopathy (DR) and Age-Related Macular Degeneration (AMD) which shows different bright spots on retina. Finally, hypothesis was developed based on region size and if only one region was classified out, it was considered as Optic Disc region. The hypothesis testing might involve parameters other than region size such as region circularity in order to improve the accuracy. Foracchia et al. [42] not only localized the ONH center as the main convergence point of blood vessels but also approximated the entire vasculature structure by fitting it with a mathematical model. The algorithm does not require accurate vasculature segmentation although it may not converge in the retinal images with low contrast ONH regions. Ying et al. [45] differentiated ONH from other bright regions, including exudates and artifacts, based on fractal dimension of blood vessels. A fractal dimension is a ratio providing a statistical index of complexity comparing how detail in a pattern changes with the scale at which it is measured. The fractal dimension of vasculature structure within $\mathrm{ONH}$ area is approximately 1.7 and it is higher as compared to fractal dimension of other bright areas. The method was applied on DRIVE database but no overall accuracy of the method was reported. Besides, the authors suspected the algorithm failure on low quality images. Park et al. [43] extracted out vasculature structure based on tensor voting. Tensor voting is the voting process of the expected candidates based on geometrical features. The image was initially contrast enhanced before application of tensor voting process. The Optic Disc was detected using mean shift procedure which points towards direction of maximum increase in the density (convergence point of blood vessels). The algorithm had higher failure rate on diseased images with white lesions. Mahfouz et al. [44] used the horizontal and vertical edge maps to locate the ONH center. These maps can be used to accurately localize the ONH center based upon a simple observation that the central retinal artery and vein emerge from the ONH mainly in the vertical direction and then progressively branch into the main horizontal vessels. The highest projection amplitude along both directions will result in center localization in a computationally efficient manner. The accuracy of the method was highly dependent on horizontal localization process.

The determination of convergence point of vasculature structure is the most efficient way to localize the Optic Disc in terms of accuracy, robustness and computational time. The methods can be applied to most of the fundus retinal images. The proved the high accuracy in case of healthy images however, the algorithms were misguided due to presence of white lesions. Retinal images diseased with Glaucoma may not have white lesions but the presence PPA may misguide the center detection of Optic Disc which is usually required for Optic Disc and Cup segmentation followed by their measurements for Glaucoma diagnosis. Therefore, combining the best features of different vasculature convergence point based algorithms may improve the center detection accuracy in the case of diseased images.

\subsection{Optic disc extraction}

The literature divides the different methods of Optic Disc Extraction in two main parts: non-model based approaches and model 
based approaches. In the non-model based approaches, the Optic Disc is extracted using different image processing algorithms such as Morphological Operations and Pixel Clustering. In the Model based approaches, the Optic Disc boundary is represented in the form of mathematical model. Both Model-based as well as Nonmodel based approaches are discussed as follows.

\subsubsection{Non-model based approaches}

For a non-model based approaches, the Optic Disc was either segmented out using different thresholding techniques or morphological operations; or approximated as an circular or elliptical area. Initially, morphological approach was introduced for Optic Disc segmentation as it required basic image processing techniques. Since the shape of Optic Disc is in the form of closed curve, therefore the structure was approximated with circular and elliptical shapes.

Walter and Klein [30] described an approach in which the RGB image was converted to Hue-Luminance-Saturation (HLS) space and a thresholding was applied to approximately localize the Optic Disc center in the luminance channel. Then the precise boundary of the Optic Disc was determined using the red channel of the RGB image via the Watershed transform [65] involving morphological pre-processing of the gradient image and region growing image segmentation. After Watershed Transform, the image is divided into small regions called superpixels. The red channel was selected as Optic Disc boundary is more prominent here. To determine the Optic Disc, the Watershed transform is constrained by markers derived from a previously calculated Optic Disc center. The identification process of the Optic Disc boundary had some problems due to blood vessels occlusion. This problem might be solved if the superpixels are generated using Simple Linear Iterative Clustering (SLIC) [66]. Nayak et al. [48] applied the morphological operations in the red channel of the RGB image in order to segment out Optic Disc. They then segmented out the Optic Cup in the green channel and calculated Cup to Disc ratio from the vertical lengths of the segmented areas. The training dataset should include larger dataset to improve generalization. Since these algorithms had the tendency to segment out the pixels other than Optic Disc therefore it led to shape approximation of the anatomical structure. Even though, Mookiah et al. [50] with their fuzzy histogram approach improved the segmentation accuracy which in their local dataset was even better than Model based Gradient Vector Flow (GVF) technique. The histogram was then correlated with roughness index to represent Optic Disc region. The authors suggested use of more diverse images and appropriate fuzzy membership functions for increase in accuracy as the algorithm over segmented PPA regions.

Zhu et al. [54] applied the Hough Transform on edge map obtained after applying morphological operations on the Luminance gray scale of the image. The Luminance grayscale can be obtained after normalizing each component of the RGB space. This method is loosely connected to main convergence point of retinal blood vessels. The authors warned about their method failure on low quality images as circular hough space might be misguided due to weak edge information. Aquino et al. [51] approximated the Optic Disc boundary using Circular Hough transform on the gradient image obtained after removal of blood vessels. The results were compared with 'Circular Gold Standards' (benchmarks) obtained from the ophthalmologists. They suspected that performance of elliptical approximation and deformable approaches could outperform circular approximation. Carmona et al. [52] obtained a set of hypothesis points that represented geometric properties and intensity levels similar to $\mathrm{ONH}$ contour pixels. Then a genetic algorithm was applied in order to find an ellipse approximating the boundary of ONH. The method was applied on DRION-DB database and the accuracy was reported in terms of pixel difference. The authors also reported its computational inefficiency and failure cases on the images having PPA. It might be improved if the hypothesis also involve statistical properties such as entropy and homogeneity which can be able to distinguish among PPA and ONH pixels.

In [55], various statistical criteria for thresholding have been employed, such as 1) percentage of higher pixel intensity values; 2) Comparative value of pixel intensity as compared to the total intensity values of the extended background image which is other than Optic Disc area. The overall accuracy was determined in terms of measuring vertical diameter of the Optic Disc. This method is applicable to both healthy images and images with AMD. Babu and Shenbagadevi [53] used $k$-means clustering after converting the image into YUV color space (one luma Y and two chrominance UV components) based on Commission Internationale de Éclairage (CIE) format. They calculated the CDR by taking the rectangular area measurements of both the Optic Disc and Optic Cup. The overall accuracy of the method was determined by comparing CDR values with those obtained from Gold Standard values from the Ophthalmologists.

In the non-model based approaches for extracting out Optic Disc, the measures such as vertical length or horizontal length were used in determining CDR after extracting out Optic Cup. CDR, although one of the important aspect in diagnosing Glaucoma, it is not crucial for Glaucoma documentation [10] as some of the patients can have large size of $\mathrm{ONH}$. Therefore it is sufficient to determine other symptoms such as neuroretinal rim loss and PPA which require exact segmentation of Optic Disc and Optic Cup. Therefore, Model based approaches can be used in determining out exact boundaries of Optic Disc and are explained in the following sections.

\subsubsection{Freeform modeling based approaches}

The model based approaches were characterized as either freeform modeling or statistical shape modeling [67]. In the freeform modeling, there is no explicit structure of template except some constraints. In this category, Active Contour Modeling (ACM) [68] has been widely investigated for extraction of Optic Disc boundaries. ACM is fundamentally a deformable contour. It changes its shape corresponding to the properties of an image based contour properties and/or knowledge based constraints. The behavior of classical parametric active contours is typically controlled by internal and external energy functions. The external energies determine the region of the model and could be derived from image features such as edges. Internal energies, such as elasticity and rigidity determine the curvature of the model and could serve as smoothness constraints to resist any deformation. The minimization of a total energy function moves the contour towards the target shape.

Mendels et al. [56,58] applied morphological operations on retinal images in YIQ(Luminance In-phase Quadrature) followed by an active contour to segment the Optic Disc. In the morphological process, a Dilation operator was first applied followed by an Erosion operator in order to retain the Disc contour whilst remove the blood vessels. Having removed the blood vessels crossing the Disc boundary, an active contour was initialized as a circle centered inside the Optic Disc. The contour was fitted to the rim of the Disc using the Gradient Vector Flow (GVF) derived from edge maps [69]. The final location of the contour was independent of initialization values. This technique was tested on nine retinal images. The morphological preprocessing has been proven effective compared to directly use of original grayscale images, however a quantitative comparison was not presented. Osareh et al. [58] applied the same method on different color spaces and found the $\mathrm{L} a b$ space to be more suitable for the application of ACM. Xu et al. [59] modified the contour energy function by defining the vector of control points with regards to smoothness, gradient orientation and median intensity. The contour was initialized using Circular Hough Transform. Moreover, the contour points were grouped into edge point clusters and uncertain point clusters using a weighted 
$k$-means algorithm where the position of the contour points were updated automatically. This operation retains the edge points close to their original positions and updates the uncertain points to their correct positions. The overall accuracy of Optic Disc extraction was high but the authors reported the algorithm failure on the images having Peripapillary Atrophy (PPA) as the energy function did not converge.

Joshi et al. [60] used a level set approach in which a contour is represented by a zero-level set of the Lipschitz function [70]. A good accuracy has been achieved based on appropriately selected initialization parameters, but the computational efficiency was low. Yu et al. made its fast implementation on the expense of accuracy by using region intensity information in level set module. The method was applied on MESSIDOR after blood vessel removal by morphological pre-processing.

ACM achieved higher accuracy in segmenting out Optic Disc as compared to Non-model based approaches however, it was prone to error when a testing image involves any textural changes or atrophy associated with any retinal diseases around the Optic Disc boundary [71]. Moreover, since these techniques were applied in their local dataset therefore their robustness is questionable. Therefore, these techniques need to be tested on public available databases in order to improve their generalization.

\subsubsection{Statistical shape modeling based approach}

Statistical shape modeling involves an offline training process in order to determine a shape model parameterizing the diverse of characteristics of the shape. In this category, ASM has been explored for the extraction of Optic Disc boundary. ASM represents shape approximation of an object using a statistic based model. The ASM deforms to reflect the boundary shape of the Optic Disc in ways that are consistent with shapes presented in the training set.

Li and Chutatape [61] proposed the use of ASM to model the boundary of Optic Disc. They differentiated the Optic Disc edges from other edges on the basis of image gradient. They compared their results with the landmarks obtained from ophthalmologists using Mean Distance to the Closest Point (MDCP). Fengshou et al. [11] used minimum Mahalanobis distance from the mean profile vector to choose the best candidate point during the local edge search. Gradient information was added as a weight into the Mahalanobis distance function. They applied their shape deformation procedure on the image composed of weighted combination of the colour channels and evaluated their results with the benchmark using other evaluation measures as well along with MDCP. We constrained the search space by defining the search boundary on the image gradient for determining the edges of Optic Disc [65]. Restricting the search space on the image improved the results as compared to original ASM. The method needs to be improved by reducing the blood vessel occlusion. Kim et al. [62] defined the imaginary circle after selecting $\mathrm{ONH}$ as brightest point. The random sample consensus technique [72] was applied in which the imaginary circle was first warped into a rectangle and then inversely warped into a circle to find the boundary of ONH. The shape warping was applied on the thresholded binary image in which boundary pixels were highly dependent on thresholding value. The author predicted more accuracy after adding constraint condition into the model selection of Random Sample Consensus (RANSAC).

The reason of Statistical shape Modeling of not achieving higher segmentation accuracy as compared to Freeform Modeling is that there were less number of parameters trained during construction of training set. The training set might include statistical measures, and even contour energy information in order to improve the segmentation accuracy. Adding prior information in the training set can be quite useful in distinguishing between PPA and Optic Disc area. Moreover, reducing blood vessel occlusion can also aid to improve segmentation accuracy.

\subsection{Optic cup extraction}

After the segmentation of Optic Disc boundary, the next step is to find out the boundary of Optic Cup in order to determine the features such as Cup to Disc Ratio and Neuroretinal Rim Loss for the diagnosis of Glaucoma. Since we know that Optic Nerve is a $3-$ Dimension structure of which the fundus image is in 2-Dimension, therefore a 3-Dimension view of the Optic Cup from stereo fundus images was obtained [59]. Similar to boundary extraction of Optic Disc, ACM was used to determine the Optic Cup boundary and they also incorporated depth analysis to minimize the energy function with regards to intensity, smoothness and shape. Automatic extraction of Optic Cup boundary using 2-Dimension fundus image have also been proposed in the literature discussed as follows.

\subsubsection{Morphology based cup segmentation}

Nayak et al. [48] used morphological operations in the green channel of RGB images to segment Optic Cup. As mentioned in Section 4.2.1, the results were used in order to classify between Normal and Glaucoma images after measuring Cup to Disc Ratio, Neuroretinal Rim Loss and vasculature shift. The classification accuracy is shown in Table 3. Babu et al. [53] applied Fuzzy C-means clustering on the Wavelet transformed green plane image after the removal of blood vessels. They compared the Cup to Disc Ratio values with those of Gold Standard obtained from the ophthalmologists. In both of the methods, the segmentation accuracy of Optic Cup was not reported

\subsubsection{Level-set approach for cup boundary detection}

Wong et al. [73] proposed a level-set approach to represent the boundary of Optic Cup in the form of gradient flow equation. The gradient flow equation was initialized by a particular threshold value. The threshold value was selected according to higher $66.7 \%$ of the normalized cumulative intensity values of the image in green channel based on the fact that Optic Cup is more prominent in Green channel. The boundary of the Optic Cup was smoothened by ellipse fitting. They applied the same approach on the red channel to obtain the Optic Disc boundary. Their later studies proposed the concept of blood vessel kinks at the edge of the Optic Cup for boundary extraction [74]. They used Canny edge detection and Wavelet transform to determine the edges of the Optic Cup. The bending of blood vessels at the angle of more than 20 degrees were empirically determined to be at the edge of Optic Cup. Joshi et al. [60] used the same information of blood vessel kinks at the edge of Optic Cup to determine the Optic Cup boundary by interpolating the vessels kinks and the Optic Cup boundary in the 3rd dimension using spline interpolation. Clinical knowledge of ophthalmologists obtained from direct 3D cup examination was utilized to approximate the cup boundary.

Up till recent, there are very limited report on Optic Cup segmentation algorithms available, and the results for the existing methods are preliminary. Since the edge between Optic Disc and Optic Cup is not usually visible in normal contrast of colour fundus images, therefore there is need to determine certain parameters which can distinguish between Optic Cup and Optic Disc. Optic Cup extraction may also require segmenting out vasculature structure and in-painting with neighborhood for accurate dimension measurement.

\subsection{Peripapillary atrophy}

In this section, we review the automatic PPA detection and segmentation methods for diagnosing Glaucoma discussed as follows:

\subsubsection{PPA localization}

Tan et al. [76] detected PPA for determining pathological myopia. They proposed the variational level set method [79] to 
extract the PPA boundary. The variational level set method is initialized with a horizontal elliptical contour within Optic Disc boundary whereas a vertical elliptical contour is initialized outside the boundary. The horizontal elliptical contour is initialized in order to reduce the influence of the edges of the retinal vessels and allow the level set function to grow and seek for the correct Optic Disc boundary. Conversely, a vertical elliptical contour is set externally as it best represents the physiological shape of the Optic Disc. The difference of these two Optic Discs is taken and thresholding in the HSV (Hue Saturation Value) color space is used to roughly segment the PPA area. They claimed the PPA localization accuracy of $95 \%$ however, they did not consider the cases if PPA is present in both temporal and nasal sides. Moreover, no PPA segmentation was done. The Test Images were taken from Singapore Cohort study of the Risk factors for Myopia (SCORM) [75].The same authors [77] proposed a fusion of two decision methods. Gray Level Covariance Matrix (GLCM) based texture analysis was used to generate a degree of roughness which is then compared for the temporal side and nasal side. The higher degree of roughness in the temporal side compared to nasal side indicates PPA presence. Statistical analysis around the Optic Disc boundary, including average intensity and standard deviation were also used to detect PPA. However, like previous method, no PPA segmentation was performed.

\subsubsection{PPA extraction}

The statistical textural features, such as contrast, correlation and variance were determined in $[78,80]$ and a feature vector of 63 features was constructed. The PPA and non-PPA pixel values were classified based on the feature vector. The training set was based on stereo images which were obtained by taking the retinal pictures of both Normal and Glaucoma patients from different angles in order make the 3D projection. The classification was performed using Linear Discriminant Analysis (LDA) [49]. The testing dataset consists of 58 Glaucoma patients out of which, 26 images have moderate to severe PPA the images with moderate to severe PPA were tested.

The segmentation of PPA is an important step not only because it is one of main indications in Glaucoma diagnosis but also it can improve the segmentation accuracy of Optic Disc if it is segmented out first. Therefore textural based properties can be used in order to classify among Optic Disc region, PPA and other retinal areas. Moreover there is need to use accurate classification algorithm which can distinguish among these regions.

\subsection{Retinal nerve fibre layer defect detection}

Several attempts had been made in order to detect RNFL based on the fact that RNFL defect detection serves as earliest signs of Glaucoma. Initially images from the modalities such as OCT or Scanning Laser Polarimetry (SLP) were used to scan the 3-Dimensional view of the retina so that RNFL was detected from these images [85-87]. Later on several Image Processing techniques were applied to detect the RNFL defect on 2-Dimensional image. Lee et al. [88] used the green plane of the fundus retinal image to detect the intensity decrease around the Optic Disc for RNFL detection. The method contributes to quantify RNFL defect and provide an objective method for Glaucoma evaluation. Hayashi et al. [81] used directional Gabor filters [89] to determine the RNFL defect. They initially removed the blood vessels and then applied the directional filter on the grayscale image. The method was prone to error as vasculature removal also erased some narrow RNFL. Moreover the false positive rate was high [90]. Prageeth et al. [82] classified the retinal images between Glaucomatous and non-Glaucomatous images using the information of RNFL defect along with CDR. They determined the RNFL defect after plotting the intensity profile of the retinal area at the edges of the retina and around the Optic Disc.
Odstrčilík et al. [83,84] analyzed the RNFL defect using Markov Random Fields (MRF). MRF is the image modeling whose parameters are estimated using Expected Maximization Algorithm [91]. The RNFL striations of Normal and Glaucomatous patients are classified on the basis of feature vector composed of MRF parameters. Despite these attempts, there is little work done to analyze the RNFL defect using 2-Dimension retinal images and the results are preliminary.

\section{Non-segmentation based classification between normal and glaucomatous retinal images}

Although there are few efforts available reflecting the classification between Normal and Glaucomatous patients based on features that do not require segmentation of retinal structures, these methods can serve as prior knowledge for measuring different Glaucomatous symptoms associated with retinal segmentation. Some of those methods are discussed as follows and their results are summarized in Table 6.

In non-segmentation based methods, Bock has major contribution $[95,96,92]$. Initially they used Pixel Intensity Values on which they applied Principal Component Analysis (PCA) [97] for dimensionality reduction. As a pre-processing step, they corrected illumination and intensity inhomogeneity then in-painted vasculature area after segmenting them out. Later on they added features such as Image Texture, FFT Coefficients, Histogram Models and B-Spline coefficients. Based on these features they calculated Glaucoma Risk Index (GRI). They used classifiers such as Naive Based Classifier, $k$-Nearest Neighbor Classifier and Support Vector Machines (SVM) [49] to classify between healthy and Glaucomatous images and they found SVM to be less prone to sparsely sampled feature space. Dua et al. [94] used Wavelet-Based Energy Features and compared the performance of different classifiers including the previously mentioned classifiers, Random Forests [98] and Sequential minimal optimization (SMO) [99]. From SMO they obtained the maximum classification accuracy.

Unlike segmentation based methods, there are few methods available based on non-segmentation analysis for the retinal images associated with Glaucoma. One of the main reason is that non-segmentation based methods are not validated by the ophthalmologists in order to distinguish among Normal and Glaucomatous images as in the case of segmentation based methods. If they are used in conjunction with segmentation based methods, they can not only be quite effective in increasing the classification accuracy among different stages of Glaucoma but also can serve as a benchmark for development of further automatic classification methods.

\section{Discussion}

Glaucoma causes changes in retinal structures which lead to peripheral vision loss if left untreated. The latest clinical information suggests observation of $\mathrm{ONH}$ area and the retinal changes around it in order to diagnose Glaucoma. Therefore after IOP measurement, the retinal exam is mostly based on measuring $\mathrm{ONH}$ related measurements such as Optic Disc size, Optic Cup size, their ratio, Neuroretinal Rim observation and PPA.

It is very difficult to determine the Optic Cup boundary from 2$D$ retinal image as there is no sharp edge indicating it. Moreover, the occlusion of blood vessels make the extraction difficult of not only Optic Cup but also Optic Disc boundary which can become even tougher if PPA is present. Also, the detection of RNFL has been quite challenging because it is not easy to distinguish between RNFL striations and vasculature structure on retina. Like fingerprint, every subject has different $\mathrm{ONH}$ structure therefore we need to have robust algorithms to determine the retinal changes related to Glaucoma diagnosis. 
Table 6

Results of different methods of non-segmentation based classification between normal and glaucomatous retinal images.

\begin{tabular}{|c|c|c|c|c|c|c|}
\hline Extraction method & Image database & Colour space & Image dimensions & Number of test images & Accuracy & Citation \\
\hline Glaucoma Risk Index & $\begin{array}{l}\text { Pattern Recognition } \\
\text { Lab Germany }\end{array}$ & 8-Bit RGB & $1600 \times 1216$ & $\begin{array}{l}336 \text { Normal and } 239 \\
\text { Glaucoma [93] }\end{array}$ & $\begin{array}{l}80 \% \text { on } 5 \text {-fold cross } \\
\text { validation (SVM) }\end{array}$ & [92] \\
\hline Wavelet Coefficients & $\begin{array}{l}\text { Kasturba Medical } \\
\text { College India }\end{array}$ & 8-Bit RGB & $560 \times 720$ & $\begin{array}{l}24 \text { Normal and } 37 \\
\text { Glaucoma }\end{array}$ & $\begin{array}{l}93 \% \text { on } 10 \text {-fold cross } \\
\text { validation (SMO) }\end{array}$ & [94] \\
\hline
\end{tabular}

The survey has been categorized into two types of algorithms i.e. segmentation based methods and non-segmentation based method. The algorithms which were applied on same datasets are grouped together so as to present their results on a common benchmark. As far as segmentation based methods are concerned, they involve series of steps; each of which can be performed using specific algorithm. It is important to observe the overall accuracy of each step from ONH localization to extraction of boundaries of Optic Disc, Optic Cup and PPA which are needed to be high to ensure the effectiveness of the overall process maintained at satisfactory level.

$\mathrm{ONH}$ localization has been the initial step in determination of Glaucoma as well as other retinal diseases such as Diabetic Retinopathy. The reason is that $\mathrm{ONH}$ has been considered the brightest region in the retinal image and therefore can serve as a way to locate other retinal structures. But, the presence of artifacts in the retinal image brighter than $\mathrm{ONH}$ led to vasculature segmentation as all the retinal blood vessels converge in $\mathrm{ONH}$. The vasculature segmentation not only localized the $\mathrm{ONH}$ accurately but also the researchers who worked on Optic Disc and Optic Cup segmentation found comparatively accurate results after segmenting out retinal blood vessels. However, the accurate segmentation of vasculature structure has itself been a challenging task.

Current vasculature segmentation methods are mostly applied and therefore quite efficient on public datasets. These datasets are composed of healthy images and the images diseased with Diabetic Retinopathy which consists of small lesions and are usually observed away of ONH. But they often segmented out PPA as well which is usually present in the images diseased with Glaucoma as shown in Fig. 7. Based on our research, textural analysis could help to distinguish among PPA and non PPA regions and incorporating them along with vasculature segmentation methods can increase their efficiency on Glaucoma images. Moreover, accurate in-painting methods by neighborhood pixels after segmenting out vasculature structure can be used to remove blood vessel occlusion problem which can lead to accurate segmentation of Optic Disc and Optic Cup. 3-D scans obtained from modalities; such as OCT, can simplify the Optic Cup boundary detection problem but these modalities are not available in every hospital. Therefore we have to limit ourselves to 2-D scans.

Apart of image processing and computer vision based techniques, pattern recognition based methods have also been applied in order to locate and extract out ONH boundary and Optic Cup. Although they were not up to the mark as they were expected; by increasing the training parameters such as texture parameters, statistical based parameters and design of accurate classifier will not only improve the results in terms of accuracy but also it will make the algorithms more robust and generalized. Huge diverse training data will significantly improve the classification efficiency. Another key issue in pattern recognition techniques is that there are few available public dataset of Glaucoma based retinal images. Although there are contributions from some of the hospitals and research labs even though, there are not enough number of retinal images diseased with Glaucoma as in the case of Diabetic Retinopathy. Since Glaucoma progresses slowly and its symptoms occur gradually, therefore it is required to develop a retinal image database of different stages of Glaucoma with annotations around different retinal structures associated with Glaucoma. The database will serve as a benchmark to test and validate algorithms related to Glaucoma based feature extraction. Detection of non-ONH anatomical structures such as RNFL can be improved by applying non-segmentation based methods on retinal images in the database. Since any of the single symptom mentioned earlier is not

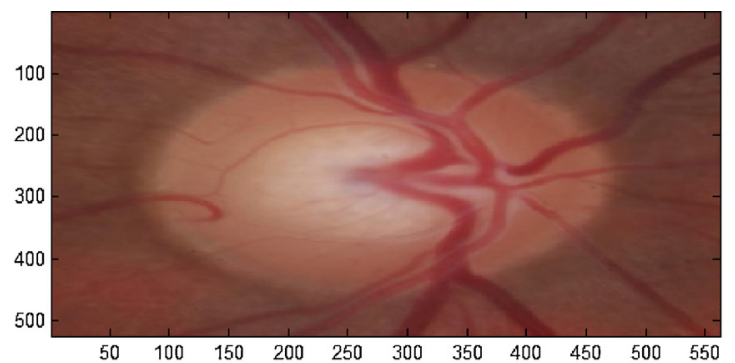

(a)

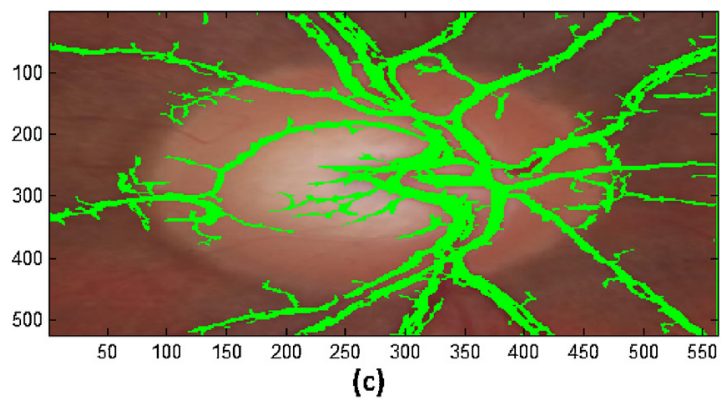

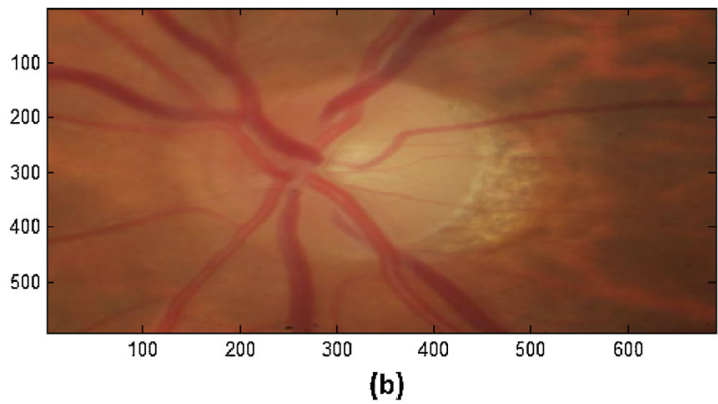

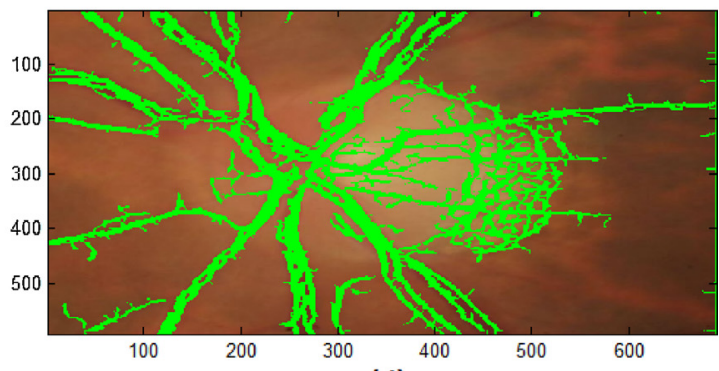

(d)

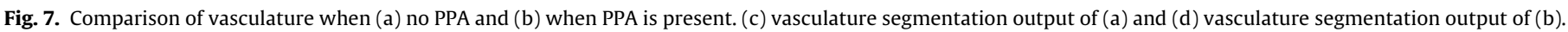


the guaranteed sign of Glaucoma, the combination of all features is required for the accurate diagnosis. If the automatic methods are improved in terms of accuracy and generalization; they will serve as a benchmark for the clinicians to diagnose the disease based on measurements obtained by using those methods.

\section{Conclusion}

Glaucoma is an evasive and dangerous eye disease and is the second leading cause of blindness globally. Since Glaucoma can not be cured, the diagnosis at their early stage can help clinicians to treat it accordingly and to prevent the patient from leading to the blindness. In this paper, we have provided a systematic review on Glaucoma, its types, current clinical methods to diagnose Glaucoma, and the methods based on automatic detection of retinal features which can be employed to assist diagnosis of Glaucoma at an early stage. The accuracy and reliability of the current clinical diagnosis are subject to inter-observer variability and every ophthalmologist determine those measurements based on their own experience. As far as retinal scans are concerned, there are significant differences among observations of different clinicians which are highly specific to the image. Moreover, 3-D scanning modalities such as OCT are not available in every hospital. Therefore, with the advent of 2-D retinal scan based automatic retinal feature methods to diagnose Glaucoma, an accurate diagnosis can be given in a time and cost effective manner.

\section{Acknowledgments}

This work is supported by EPSRC-DHPA funded project "Automatic Detection of Features in Retinal Imaging to Improve Diagnosis of Eye Diseases". We would like to thank reviewers who provided detailed and constructive comments on an earlier version of this paper.

\section{Appendix A. Evaluation functions}

\section{A.1. Overlapping Score}

The Overlapping Score evaluates the degree of overlap of two regions and is used to determine the extent to which the segmented objects match. The Overlapping Score can be defined as in equation A.1:

$S(A, B)=\frac{|A \cap B|}{|A \cup B|}$

where $A$ and $B$ are the segmented images surrounded by model boundary and annotations from the ophthalmologists respectively, $\cap$ denotes the intersection and $\cup$ denotes the union. Its value varies between 0 and 1 where a higher value, indicates an increased degree of overlap.

\section{A.2. Mean Absolute Difference}

The Mean Absolute Difference (MAD) is the mean difference between the extracted boundary and the average boundary obtained from the ophthalmologists as shown below:

textrmMAD $=\frac{1}{n} \sum_{i=1}^{n} \sqrt{e_{x_{i}}^{2}+e_{y_{i}}^{2}}$,

where $e_{x_{i}}$ and $e_{y_{i}}$ are the error values at the particular point.

\section{A.3. Correlation Coefficient}

The Correlation Coefficient is defined as:

$\rho_{X, Y}=\frac{E(X Y)-E(X) E(Y)}{\sqrt{E\left(X^{2}\right)-(E(X))^{2}} \sqrt{E\left(Y^{2}\right)-(E(Y))^{2}}}$,

where $X$ and $Y$ represent the datasets whereas $E()$ defines mean of dataset.

\section{Appendix B. Public databases used for experimentation}

A brief explanation of the public databases used in the literature review are explained as in this section. Most of the public databases were constructed for automatic feature extraction of Diabetic Retinopathy but they were used for extraction of anatomical structures such as Optic Disc used in Glaucoma diagnosis. Some of the public databases were constructed particularly for automatic feature extraction of Glaucoma.

\section{B.1. STARE (STructured Analysis of the REtina)}

The STARE database [34] is the part of STARE project initiated by Michael Goldbaum at University of California, San Diego in 1975 for the analysis of retinal images. It is being funded by National Institute of Health, USA since 1986. Uptil now, the database is composed of 81 in which 31 of them are from healthy retinas and 50 images show the symtoms of the retinal diseases such as exudates and haemorrhages that completely obscure the Optic Nerve Head. All these images were acquired using a TopCon TRV-50 fundus camera at $35^{\circ}$ field-of-view, and subsequently digitized at $605 \times 700$ pixels in resolution, 24 bits per pixel.

\section{B.2. DRIVE (Digital Retinal Images for Vessel Extraction)}

The photographs for the DRIVE database [36] were obtained from a diabetic retinopathy screening program in The Netherlands. The screening population consisted of 400 diabetic subjects between $25-90$ years of age. 40 photographs have been randomly selected, 33 do not show any sign of diabetic retinopathy and 7 show signs of mild early diabetic retinopathy. The images were acquired using a Canon CR5 non-mydriatic (which does not cause dilation to eye pupil) camera with a $45^{\circ}$ field of view (FOV). Each image was captured using 8-bits per color plane at 768 by 584 pixels. Each image has the manual segmentation of the vasculature structure.

\section{B.3. DIARETDBO (Standard Diabetic Retinopathy Database Calibration Level 0)}

This is a public database [100] for benchmarking diabetic retinopathy detection from digital images. It consists of 130 color fundus images of which 20 are Normal and 110 contain signs of the diabetic retinopathy (hard exudates, soft exudates, micronaneuyrysms, hemorrhages and neovascularization). Images, obtained from Kuopio University Hospital, were captured with a $50^{\circ}$ field-of-view digital fundus camera at $1500 \times 1152$ pixels. The dataset can be used to evaluate the general performance of diagnostic methods. This dataset is referred to as "calibration level 0 fundus images" as it corresponds to the situation where no calibration is performed and the imaging was performed with unknown amount of imaging parameters. 


\section{B.4. DIARETDB1 (Standard Diabetic Retinopathy Database Calibration Level 1)}

The images of the database [101] are obtained from same hospital and used for benchmarking diabetic retinopathy. It consists of 89 colour fundus images of which 84 contain at least mild nonproliferative signs (Microaneurysms) of the diabetic retinopathy, and 5 are considered as Normal which do not contain any signs of the diabetic retinopathy according to all experts who participated in the evaluation. Images were captured using the same $50^{\circ}$ fieldof-view digital fundus camera at $1500 \times 1152$ with varying imaging settings. This data set is referred to as "calibration level 1 fundus images" as the images are captured after encountering different amounts imaging noise and optical aberrations.

\section{B.5. MESSIDOR (Méthodes d'Evaluation de Systèmes de Segmentation et d'Indexation Dédiées à l'Ophtalmologie Rétinienne)}

MESSIDOR [46] is a project funded by the French Ministry of Research and Defense within a 2004 TECHNO-VISION program for implementing and testing segmentation algorithms. It contains 1200 eye fundus color images of the posterior pole acquired by the Hôpital Lariboisière Paris, the Faculté de Médecine St. Etienne and the LaTIM-CHU de Brest (France). 800 of these images were captured with pupil dilation (one drop of Tropicamide at 10\%) and 400 without dilation, using a Topcon TRC NW6 non-mydriatic retinograph with a $45^{\circ}$ field of view. The images are $1440 \times 960$, $2240 \times 1488$, or $2304 \times 1536$ pixels in size and 8 -bits per color plane and are provided in TIFF format. 540 images are from patients not affected by Diabetic Retinopathy and 660 images correspond to patients affected by the illness. To prevent the inclusion of any kind of skew, although some images are not suitable for processing (i.e., images too blurred or with severe enough cataract), no exclusion criteria was applied. To make evaluation of the algorithm performance on this database possible, the Optic Disc rim was manually delimited by experts producing by this way a gold standard set.

\section{B.6. DRION-DB (Digital Retinal Images for Optic Nerve Segmentation Database)}

This database [52] is composed by joint collaboration of three Spanish organizations, i.e. Universidad Complutense, Hospital Miguel Servet and Universidad Nacional de Educación a Distancia. It has 110 retinal images with each image having the resolution of $600 \times 400$ pixels and the Optic Disc annotated by two experts with 36 landmarks. The images were acquired with a colour analogical fundus camera, approximately centered on the ONH and they were stored in slide format. In order to have the images in digital format, they were digitized using a HP-PhotoSmart-S20 high-resolution scanner, RGB format, resolution $600 \times 400$ and 8-bits/pixel. Independent contours from 2 medical experts were collected by using a software tool provided for image annotation. A person with medical education and solid experience in ophthalmology was considered as an expert. In each image, each expert traced the contour by selecting the most significant papillary contour points and the annotation tool connected automatically adjacent points by a curve.

\section{B.7. RIM-ONE (An Open Retinal Image Database for Optic Nerve Evaluation)}

RIM-ONE [102] is exclusively focused on ONH segmentation, it has 169 high-resolution images and 5 manual reference segmentations and a gold standard of each one. The high number of expert segmentations enables the creation of reliable gold standards and the development of high accurate segmentation algorithms. The designed database is composed of $169 \mathrm{ONH}$ images obtained from 169 full fundus images of different subjects. These retinographs have been captured in the three hospitals cited before which are located in different Spanish regions. Compiling images from different medical sources guarantee the acquisition of a representative and heterogeneous image set. All the retinographs are non mydriatic retinal photographs captured with specific flash intensities, avoiding saturation. The images are classified in different subsets, as domain experts have indicated:

- Normal eye (non-glaucomatous): 118 images.

- Early glaucoma: 12 images.

- Moderate glaucoma: 14 images.

- Deep glaucoma: 14 images.

- Ocular hypertension (OHT): 11 images

\section{B.8. ORIGA-light (An Online Retinal Fundus Image Database for Glaucoma Analysis and Research)}

The database [63] is based on retinal images collected in a population based study, Singapore Malay Eye Study. It contains 650 annotated retinal images. Each image is tagged with grading information of different features related to Glaucoma. This database is not available publically now.

\section{References}

[1] Roodhooft J. Leading causes of blindness worldwide. Bull Soc Belge Ophtalmol 2002;283:19-25

[2] Suzanne R. The most common causes of blindness; 2011 http://www. livestrong.com/article/92727-common-causes-blindness

[3] Michelson G, Hornegger J, Wärntges S, Lausen B. The papilla as screening parameter for early diagnosis of glaucoma. Deutsches Arzteblatt International $105 ; 2008$.

[4] Types of glaucoma; May 2011 http://www.glaucoma.org/glaucoma/types-ofglaucoma.php

[5] Weinreb RN, Khaw PT. Primary open-angle glaucoma. The Lancet 2004;363:1711-20.

[6] Reus TGJN, Lemij H. Estimating the clinical usefulness of optic disc biometry for detecting glaucomatous change over time. Eye 2006;20:755-63.

[7] Liu J, Lim JH, Wong WK, Li H, Wong TY. Automatic cup to disc ratio measurement system (04 2011); 2011 http://www.faqs.org/ patents/app/20110091083

[8] Jonas J, Fernández M, Stürmer J. Pattern of glaucomatous neuroretinal rim loss. Ophthalmology 1993;100:63-8.

[9] Harizman N, Oliveira C, Chiang A, Tello C, Marmor M, Ritch R, et al. The isnt rule and differentiation of normal from glaucomatous eyes. Ophthalmology 2006;124:1579-83.

[10] Jonas J, Budde W, Jonas S. Ophthalmoscopic evaluation of optic nerve head Survey of Ophthalmology 1999;43:293-320.

[11] Fengshou Y. Extraction of features from fundus images for glaucoma assess ment, Master's thesis. National University of Singapore; 2011.

[12] Maclver S, Sherman M, Slotnick S, Sherman J. Comparison of optos p200 and p200dx, Tech. rep.; 2011.

[13] Jonas J. Clinical implications of peripapillary atrophy in glaucoma. Current Opinion in Ophthalmology 2005;16:84-8

[14] Jonas J, Fernández M, Naumann GOH. Glaucomatous parapapillary atrophyoccurrence and correlations. Ophthalmology 1992;110:214-22.

[15] Ehrlich JR, Radcliffe NM. The role of clinical parapapillary atrophy evaluation in the diagnosis of open angle glaucoma. Clinical Ophthalmology 2010;4:971-6.

[16] Teng CC, Moraes CGVD, Prata vS, Tello C, Ritch R, Liebmann JM. $\beta$-Zone parapapillary atrophy and the velocity of glaucoma progression. Ophthalmology $117 ; 2010$.

[17] Diagnosis and management of chronic open angle glaucoma and ocular hypertension, Tech. rep. National Collaborating Centre for Acute Care; 2009

[18] Diagnosing and treating glaucoma and raised eye pressure, Tech. rep. National Institute of Health and Clinical Excellence (NICE); 2009.

[19] Barnett T. Goldmann applanation tonometry; September 2000 http://webeye ophth.uiowa.edu

[20] Glaucoma diagnosis: the role of optic nerve examination, Tech. rep. Rotterdam Eye Hospital; 2007.

[21] Drake MV. The importance of corneal thickness; April 2011 http://www.glaucoma.org

[22] Spry P, Johnson C, McKendrick A, Turpin A. Measurement error of visual field tests in glaucoma. British Journal of Ophthalmology 2003;87:107-12. 
[23] Fasih U, Shaikh A, Shaikh N, Fehmi M, Jafri AR, Rahman A. Measurement error of visual field tests in glaucoma. Pakistan Journal of Ophthalmology 2009;25:145-51.

[24] Schuman JS. Spectral domain optical coherence tomography for glaucoma; 2008.

[25] Sitaraman C, Sharma K, Sahai A, Gangwal W. Analysis of rnfl thickness in normal, ocular hypertensives and glaucomatous eyes with oct. In: Proceedings of All India Ophthalmological Society (AIOS). 2009. p. 246.

[26] Kotowski J, Wollstein G, Folio LS, Ishikawa H, Schuman JS. Clinical use of oct in assessing glaucoma progression, Ophthalmic Surgery. Laser and Imaging 2011;42:S6-14

[27] Chaudhuri S, Chatterjee S, Katz N, Nelson M, Goldbaum M. Automatic detection of the optic nerve head in retinal images. In: Proceedings of the IEEE International Conference on Image Processing, Vol. 1. 1989.

[28] Sinthanayothin C, Boyce J, Cook H, Williamson T. Automated localisation of the optic disc, fovea, and retinal blood vessels from digital colour fundus images. British Journal of Ophthalmology 1999;83:902-10.

[29] Lowell J, Hunter A, Steel D, Basu A, Ryder R, Fletcher E, et al. Optic nerve head segmentation. IEEE Transactions on Biomedical Engineering 2004;23:256-64.

[30] Walter T, Klein J-C. Segmentation of color fundus images of the human retina: Detection of the optic disc and the vascular tree using morphological techniques. In: Proceedings of the Second International Symposium on Medical Data Analysis. 2001. p. 282-7.

[31] Sekhar S, Al-Nuaimy W, Nandi A. Automated localisation of retinal optic disk using hough transform. In: Proceedings of 5th IEEE International Symposium on Biomedical Imaging: From Nano to Macro. 2008. p. 1577-80.

[32] Gonzalez RC, Woods RE, editors. Digital image processing. 3rd ed. Upper Saddle River, NJ, USA: Prentice Hall; 2006.

[33] Rizon M, Yazid H, Saad P, Shakaff AYM, Saad AR. Object detection using circular Hough transform. American Journal of Applied Sciences 2;2005.

[34] Goldbaum M. Structured analysis of the retina (stare); 2000 http://www.ces.clemson.edu/ahoover/stare/

[35] Haar Ft. Automatic localization of the optic disc in digital colour images of the human retina, Master's thesis. Utrecht University; 2005.

[36] Staal J, Abramoff M, Niemeijer M, Viergever M, Ginneken Bv. Ridge based vessel segmentation in color images of the retina. IEEE Transactions on Medical Imaging 23;2004.

[37] Osareh A, Mirmehdi M, Thomas BT, Markham R. Classification and localisation of diabetic-related eye disease. In: Proceedings of 7th European conference on computer vision-Part IV, Vol. 2. 2002. p. 1625-9.

[38] Lalonde M, Beaulieu M, Gagnon L. Fast and robust optic disc detection using pyramidal decomposition and hausdorff-based template matching. IEEE Transactions on Medical Imaging 2001;20:1193-200.

[39] Youssif AA-HA-R, Ghalwash AZ, Ghoneim AASA-R. Optic disc detection from normalized digital fundus images by means of a vessels direction matched filter. IEEE Transactions on Medical Imaging 2008;27:11-8.

[40] Li H, Chutatape O. Automatic location of optic disk in retinal images. In: Proceedings of International Conference on Image Processing. 2001. p. $837-40$.

[41] Hoover A, Goldbaum M. Locating the optic nerve in a retinal image using the fuzzy convergence of the blood vessels. IEEE Transactions on Medical Imaging 2003;22:951-8

[42] Foracchia M, Grisan E, Ruggeri A. Detection of optic disc in retinal images by means of a geometrical model of vessel structure. IEEE Transactions on Medical Imaging 2004;23:1189-95.

[43] Park J, Kien NT, Lee G. Optic disc detection in retinal images using tensor voting and adaptive mean-shift. In: IEEE International Conference on Intelligent Computer Communication and Processing. 2007. p. 237-41.

[44] Mahfouz A, Fahmy AS. Fast localization of the optic disc using projection of image features. IEEE Transactions on Image Processing 2010;19:3285-9.

[45] Ying H, Zhang M, Liu J-C. Fractal-based automatic localization and segmentation of optic disc in retinal images. In: Proceedings of the 29th Annual International Conference of the IEEE EMBS. 2007. p. 4139-41.

[46] (méthodes d'evaluation de systèmes de segmentation et d'indexation dédiées à l'ophtalmologie rétinienne); 2008 http://messidor.crihan. fr/description-en.php

[47] Yu H, Barriga S, Agurto C, Echegaray S, Pattichis M, Zamora G, et al. Fast localization of optic disc and fovea in retinal images for eye disease screening. In: Proceedings of SPIE conference on medical imaging 2011: computer-aided diagnosis. 2011.

[48] Nayak J, Acharya R, Bhat P, Shetty N, Lim T-C. Automated diagnosis of glaucoma using digital fundus images. Journal of Medical Systems 2009;33:337-46.

[49] Smola A, Vishwanathan S. Introduction to Machine Learning. Cambridge, United Kingdom: Cambridge University Press; 2008.

[50] Mookiah MRK, Acharya UR, Chua CK, Min LC, Ng EYK, Mushrif MM, et al. Automated detection of optic disk in retinal fundus images using intuitionistic fuzzy histon segmentation. Journal of Engineering in Medicine 2012;227:37-49.

[51] Aquino A, Gegúndez-Arias M, Marín D. Detecting the optic disc boundary in digital fundus images using morphological, edge detection, and feature extraction techniques. IEEE Transactions on Medical Imaging 2010;29:1860-9.

[52] Carmona E, Rincón M, García-Feijoo J, Martínez-de-la Casa J. Identification of the optic nerve head with genetic algorithms. Artificial Intelligence in Medicine 2008;43:243-59.
[53] Babu TRG, Shenbagadevi S. Automatic detection of glaucoma using fundus image. European Journal of Scientific Research 2011;59:22-32.

[54] Zhu X, Rangayyan RM, Ells AL. Detection of the optic nerve head in fundus images of the retina using the hough transform for circles. Journal of Digital Imaging 2010;23:332-41.

[55] Kose C, Ikibas C. Statistical techniques for detection of optic disc and macula and parameters measurement in retinal fundus images. Journal of Medical and Biological Engineering 2010;31:395-404.

[56] Mendels F, Heneghan C, Harper P, Reilly R, Thiran J. Extraction of the optic disk boundary in digital fundus images. In: Proceedings of the First BMES / EMBS Conference. 1999. p. 1139.

[58] Mendels F, Heneghan C, Thiran J. Identification of the optic disk boundary in retinal images using active contours. In: Proceedings of Irish Machine Vision and Image Processing Conference (IMVIP). 1999. p. 103-15.

[58] Osareh A, Mirmehdi M, Thomas B, Markham R. Comparison of colour spaces for optic disc localisation in retinal images. In: Proceedings of the 16th International Conference on Pattern Recognition. 2002. p. 743-6.

[59] Xu J, Chutatape O, Sung E, Zheng C, Kuan PCT. Optic disk feature extraction via modi?ed deformable model technique for glaucoma analysis. Pattern Recognition 2005;40:2063-76.

[60] Joshi G, Sivaswamy J, Krishnadas S. Optic disk and cup segmentation from monocular color retinal images for glaucoma assessment. IEEE Transactions on Medical Imaging 2011;30:1192-205.

[61] Li H, Chutatape O. Boundary detection of optic disk by a modified asm method. Pattern Recognition 2003;36:2093-104.

[62] Kim SK, Kong H-J, Seo J-M, Cho BJ, Park KH, Hwang JM, et al. Segmentation of optic nerve head using warping and ransac. In: Proceedings of the 29th Annual International Conference of the IEEE EMBS. 2007. p. 900-3.

[63] Zhang Z, Yin F, Liu J, Wong W, Tan N, Lee B, et al. Origa(-light): an online retinal fundus image database for glaucoma analysis and research. In: IEEE Annual International Conference of Engineering in Medicine and Biology Society (EMBC). 2010. p. 3065-8.

[65] Haleem MS, Han L, Li B, Nisbet A, Hemert Jv, Verhoek M. Automatic extraction of optic disc boundary for detecting retinal diseases. In: 14th IASTED International Conference on Computer Graphics and Imaging (CGIM). 2013.

[65] Roerdink JBTM, Meijster A. The watershed transform: de nitions, algorithms and parallelization strategies. Fundamenta Informaticae 2001;41:187-228.

[66] Achanta R, Shaji A, Smith K, Lucchi A, Fua P, Süsstrunk S. Slic superpixels compared to state-of-the-art superpixel methods. IEEE Transactions on Pattern Analysis and Machine Intelligence 34;2011.

[67] Mclnerney T, Terzopoulos D. Deformable models in medical image analysis: a survey. Medical Image Analysis 1;1996.

[68] Kass M, Witkin A, Terzopoulos D. Snakes active contour models. International Journal of Computer Vision 1;1987.

[69] Xu C, Prince J. Snakes, shapes, and gradient vector flow. IEEE Transactions on Image Processing 7;1998.

[70] Ricco L. Lipschitz functions, Tech. rep.; 2004

[71] Kande G, Subbaiah P, Savithri T. Feature extraction in digital fundus images. Medical Image Analysis 29;2009.

[72] Fischler M, Bolles R. Random sample consensus: a paradigm for model fitting with applications to image analysis and automated cartography. Communications of the ACM 1981;24:381-95.

[73] Wong D, Liu J, Lim J, Jia X, Yin F, Li H, et al. Level-set based automatic cup-todisc ratio determination using retinal fundus images in argali. In: 30th Annual international conference of the IEEE engineering in medicine and biology society. 2008. p. 2266-9.

[74] Wong D, Liu J, Lim J, Jia X, Yin F, Li H, et al. Automated detection of kinks from blood vessels for optic cup segmentation in retinal images. Proceedings of the SPIE Medical Imaging 2009;7260, 72601J-172601J-8.

[75] Saw S-M, Shankar A, Tan S-B, Taylor H, Tan DTH, Stone RA, et al. A cohort study of incident myopia in singaporean children. Investigative Ophthalmology and Visual Science 2006;47:1839-44.

[76] Tan N, Liu J, Lim J, Zhang Z, Lu S, Li H, et al. Automatic detection of pathological myopia using variational level set. In: 31st Annual International Conference of the IEEE Engineering in Medicine and Biology Society. 2009. p. 3609-12.

[77] Lee B, Wong D, Tan N, Zhang Z, Lim J, Li H, et al. Fusion of pixel and texture features to detect pathological myopia. In: 5th IEEE Conference on Industrial Electronics and Applications (ICIEA). 2010. p. 2039-42.

[78] Muramatsu C, Hatanaka Y, Sawada A, Yamamoto T, Fujita H. Computerized detection of peripapillary chorioretinal atrophy by texture analysis. In: Conference Proceedings IEEE Engineering in Medicine and Biology Society. 2011. p. 5947-50.

[79] Mitiche A, Ayed IB, editors. Variational and level set methods in image segmentation. London, United Kingdom: Springer Topics in Signal Processing; 2011.

[80] Muramatsu C, Nakagawa T, Sawada A, Hatanaka Y, Hara T, Yamamoto T, et al. Automated segmentation of optic disc region on retinal fundus photographs: Comparison of contour modeling and pixel classification methods. IEEE Transactions on Medical Imaging 2011;101:23-32.

[81] Hayashi Y, Nakagawa T, Hatanaka Y, Aoyama A, Kakogawa M, Hara T, et al. Detection of retinal nerve fiber layer defects in retinal fundus images using gabor filtering. Proceedings of the SPIE Medical Imaging 2007;6514, 65142 Z-1-65142 Z-8.

[82] Prageeth P, David J, Kumar A. Early detection of retinal nerve fiber layer defects using fundus image processing. In: IEEE Conference of Recent Advances in Intelligent Computational Systems (RAICS). 2011. p. 930-6. 
[83] Odstrčilík J, Kolář R, Harabiš V, Gazárek J, Jan J. Retinal nerve fiber layer analysis via markov random fields texture modelling. In: 18th European Signal Processing Conference. 2010. p. 1650-4.

[84] Odstrčilík J, Kolář R, Jan J, Gazárek J, Kuna Z, Vodakova M. Analysis of retinal nerve fiber layer via markov random fields in color fundus images. In: 19th International Conference on Systems, Signals and Image Processing (IWSSIP). 2012. p. 504-7.

[85] Peli E, Hedges T, Schwartz B. Computer measurement of retinal nerve fiber layer striations. Applied Optics 1989;28:1128-34.

[86] Vermeer K, Vos F, Lemij H, Vossepoel A. Detecting wedge shaped defects in polarimetric images of the retinal nerve fiber layer. In: Proceedings of MICCAI '02 Proceedings of the 5th International Conference on Medical Image Computing and Computer-Assisted Intervention-Part I. 2002.

[87] Lin S, Singh K, Jampel H. Optic nerve head and retinal nerve fiber layer analysis: A report by the american academy of ophthalmology. Ophthalmology 2008; 115:1937-49.

[88] Lee S, Kim K, Seo J, Kim D, Chung H, Park K, et al. Automated quantification of retinal nerve fiber layer atrophy in fundus photograph. In: 26th Annual International Conference of the IEEE Engineering in Medicine and Biology Society. 2004. p. 1241-3.

[89] Chao W-l. Gabor wavelet transform and its application, Tech. rep.; 2010.

[90] Muramatsu C, Hayashi Y, Sawada A, Hatanaka Y, Hara T, Yamamoto T, et al. Detection of retinal nerve fiber layer defects on retinal fundus images for early diagnosis of glaucoma. Journal of Biomedical Optics 2010;15:1-7.

[91] Borman S. The expectation maximization algorithm: a short tutorial, Tech. rep.; 2004.

[92] Bock R, Meier J, Nyúl L, Hornegger J, Michelson G. Glaucoma risk index:automated glaucoma detection from color fundus images. Medical Image Analysis 2010;14:471-81.

[93] Köhler T, Budai A, Kraus M, Odstrcilik J, Michelson G, Hornegger J. Automatic no-reference quality assessment for retinal fundus images using vessel segmentation. In: 26th IEEE international symposium on computer-based medical systems. 2013. p. 2013.

[94] Dua S, Acharya UR, Chowriappa P, Sree SV. Wavelet-based energy features for glaucomatous image classification. IEEE Transactions on Information Technology in Biomedicine 2012;16:80-7.

[95] Meier J, Bock R, Michelson G, Nyúl L, Hornegger J. Effects of preprocessing eye fundus images on appearance based glaucoma classification. In: Proceedings of the 12th international conference on Computer analysis of images and patterns, Vol. 4673. 2007. p. 165-72.

[96] Bock R, Meier J, Michelson G, Nyúl L, Hornegger J. Classifying glaucoma with image-based features from fundus photographs. In: Proceedings of the 29th DAGM (German conference on pattern recognition) conference on pattern recognition, Vol. 4713. 2007. p. 355-64.

[97] Zhao W, Chellappa R, Phillips P, Rosenfield A. Face recognition: A literature survey. ACM Computer Survey 2003:35:399-458.

[98] Breiman L. Random forests, Tech. rep. Berkeley: University of California; 2001.

[99] Keerthi S, Shevade S, Bhattacharyya C, Murthy K. Improvements to Platt's SMO algorithm for SVM classifier design, Tech. rep. National University of Singapore; 1998.

[100] Kauppi T, Kalesnykiene V, Kamarainen J, Lensu L, Sorri I, Uusitalo H, et al. Diaretdb Evaluation database and methodology for diabetic retinopathy algorithms, Tech. rep.; 2006.
[101] Kauppi T, Kalesnykiene V, Kamarainen J, Lensu L, Sorri I, Raninen A, et al. Diaretdb1 diabetic retinopathy database and evaluation protocol, Tech. rep. 2007.

[102] Fumero F, Alayon S, Sanchez J, Sigut J, Gonzalez-Hernandez M. Rim-one. An open retinal image database for optic nerve evaluation. In: Proceedings of the 24 th international symposium on computer-based medical systems (CBMS 2011), 2011. 2011.

Muhammad Salman Haleem received B.Engg. in electronic engineering from NED University of Engineering and Technology, Pakistan in 2008. He attained M.S. in elec trical engineering from Illinois Institute of Technology, Chicago, IL, USA (2011). He is currently a PhD research student at Manchester Metropolitan University, UK. The title of his thesis is "Automatic Detection of Features to Assist Diagnosis of Retina Diseases". For this project, he received the prestigious Dorothy Hodgkin Postgraduate Award (DHPA) from the Engineering and Physical Sciences Research Counci (EPSRC). His areas of interest are Computer Vision, Image Processing, Machine Learning and Data mining.

Liangxiu Han is currently a reader at the School of Computing, Mathematics and Digital Technology, Manchester Metropolitan University. His research areas mainly lie in the development of novel architectures for large-scale networked distributed systems (e.g. Cloud/Grid/Service-oriented computing/Internet) large-scale data mining (application domains include web mining, biomedical images, environmental sensor data, network traffic data, cyber security, etc.), and knowledge engineering. As a PI or Co-PI, his research is funded by research councils, industries and charity bodies, in the research areas mentioned above.

Jano van Hemert has a PhD in mathematics and physical sciences from Leiden University, The Netherlands (2002). He is the imaging research manager and academic liaison at Optos; an innovative retinal imaging company with a vision to be the leading provider of retinal diagnostics. Since 2010, he is an honorary fellow of the University of Edinburgh. Since 2011, he is a member of The Young Academy of The Royal Society of Edinburgh. From 2007 until 2010 he led the research of the UK National e-Science Centre, supported by an EPSRC Platform Grant. He has held research positions at the Leiden University (NL), the Vienna University of Technology (AT) and the National Research Institute for Mathematics and Computer Science (NL). In 2004, he was awarded the Talented Young Researcher Fellowship by the Netherlands Organization for Scientific Research. In 2009, he was recognised as a promising young research leader with a Scottish Crucible.

Baihua Li received B.Sc. and M.Sc. degrees in electronic engineering from Tianjin University, China and Ph.D. degree in computer science from Aberystwyth University, UK. She is a senior lecturer in the School of Computing, Mathematics \& Digita Technology, Manchester Metropolitan University, UK. Her current research interest include computer vision, image processing pattern recognition, advanced compute graphics, human motion analysis and behavior understanding from multi-modality imaging and sensory data. About 40 fully refereed research papers have been published in leading national/international journals and conferences, including IEEE Transactions, PR and IVC. She takes a role as reviewer and Program Committee member for a number of high quality journals and conferences. She is a member of the BMVA. 